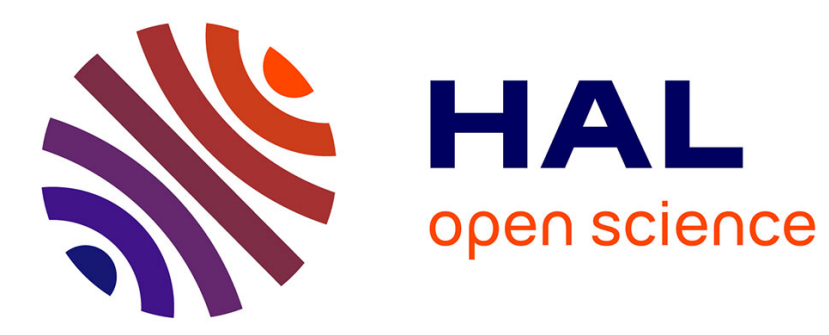

\title{
Direct simulations for wall modeling of multicomponent reacting compressible turbulent flows
}

\author{
Olivier Cabrit, Franck Nicoud
}

\section{To cite this version:}

Olivier Cabrit, Franck Nicoud. Direct simulations for wall modeling of multicomponent reacting compressible turbulent flows. Physics of Fluids, 2009, 21 (5), pp.055108. 10.1063/1.3123528 . hal00803823

\section{HAL Id: hal-00803823 \\ https://hal.science/hal-00803823}

Submitted on 22 Mar 2013

HAL is a multi-disciplinary open access archive for the deposit and dissemination of scientific research documents, whether they are published or not. The documents may come from teaching and research institutions in France or abroad, or from public or private research centers.
L'archive ouverte pluridisciplinaire HAL, est destinée au dépôt et à la diffusion de documents scientifiques de niveau recherche, publiés ou non, émanant des établissements d'enseignement et de recherche français ou étrangers, des laboratoires publics ou privés. 
published in Physics of Fluids, 21, 055108 (2009)

\title{
Direct simulations for wall modeling of multicomponent reacting compressible turbulent flows
}

\author{
Olivier Cabrit* \\ Centre Européen de Recherche et de Formation \\ Avancée en Calcul Scientifique (CERFACS), \\ 42 avenue Gaspard Coriolis, \\ 31057 Toulouse, France
}

\author{
Franck Nicoud \\ Université Montpellier 2, \\ UMR CNRS 5149 / CC51, \\ Place Eugène Bataillon, \\ 34095 Montpellier, France
}

(Dated: May 28, 2009) 


\begin{abstract}
A study of multicomponent reacting channel flows with significant heat transfer and low Mach number has been performed using a set of direct and wall-resolved large eddy simulations. The Reynolds number based on the channel half-height and the mean friction velocity is $R e_{\tau}=300$ for DNS, and $R e_{\tau}=1000$ for wall-resolved LES. Two temperature ratios based on the mean centerline temperature, $T_{c}$, and the temperature at the wall, $T_{w}$, are investigated: $T_{c} / T_{w}=1.1$ for DNS, and $T_{c} / T_{w}=3$ for wall-resolved LES. The mass/momentum/energy balances are investigated, specially showing the changes induced by multicomponent terms of the Navier-Stokes equations. Concerning the flow dynamics, the data support the validity of the Van Driest transformation for compressible reacting flows. Concerning heat transfer, two multicomponent terms arise in the energy conservation balance: the laminar species diffusion which appears to be negligible in the turbulent core, and the turbulent flux of chemical enthalpy which cannot be neglected. The data also show that the mean composition of the mixture is at equilibrium state; a model for the turbulent flux of chemical enthalpy is then proposed and validated. Finally, models for the total shear stress and the total heat flux are formulated and integrated in the wall normal direction to retrieve an analytical law-of-the-wall. This wall model is tested favorably against the DNS/LES database.
\end{abstract}

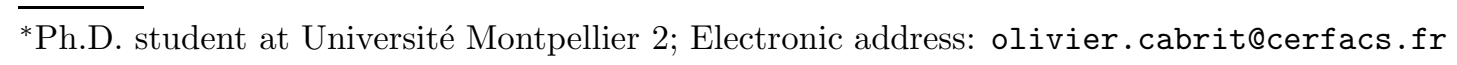




\section{INTRODUCTION}

In many industrial applications, the thermodynamic conditions are severe so that momentum and energy transfers are strongly coupled. Foreseeing the near wall region behavior then becomes an issue because it is affected by the variations in fluid properties and physical effects usually not present in academic cases. For instance, the design of devices such as piston engines or rocket-motor nozzles requires the understanding and modeling of the multicomponent reacting compressible turbulent boundary layer. Classical wall models are clearly not appropriate for this flow configuration and their improvement is necessary. From a general point of view the physical effects leading to modeling issues can be listed as follows: compressibility (generated either by strong temperature gradients or high Mach number), wall heat transfer, streamwise pressure gradient, surface roughness, mass transfer at the wall (associated to blowing and suction effects), buoyancy, and chemical reactions. The present study focuses on the case when chemical reactions, wall heat transfer, and compressibility effects are present and potentially interact. Let's start by taking a look at a non-exhaustive overview of the historical and theoretical bases of the present study, distinguishing works that aim at improving the understanding of wall turbulence structure from those who deal with its modeling.

In his pioneer work[1], Prandtl is the first one who clarified the understanding of NavierStokes equations by neglecting inertia terms near the wall. He gave the name "transition layer" to what we now call the boundary layer. Blasius[2], von Kármán[3, 4], Clauser[5] and many others extended the boundary layer theory leading their studies to the derivation of the usual log-law formula for turbulent flows. Because this approximation is only justified for zero pressure gradient incompressible boundary layers, the need of taking care of others physical effects quickly arose. For instance, Patankar and Spalding[6] developed a set of formula that aimed at accounting for the modifications induced by pressure gradient and mass transfer through the wall. Concerning strong temperature gradients and Mach number effects, in the 1950's through the mid 1970's, many experimental studies focussed on the assessment of global quantities at the wall (friction coefficient, Nusselt number) and some empirical correlations of engineering interest were derived[7]. In the 1980's, the assessment of temperature profiles has received a major advance by the work of Kader[8] who derived an efficient correlation to account for Prandtl number effects. Despite these studies, the models 
used in design codes for industrial applications remained simple log-law type formulations even if some important physical effects were missing.

The compressible turbulent boundary layer with or without heat transfer is now well documented[9-11] and the advent of direct numerical simulations (DNS)[12-15] and wellresolved large-eddy simulations (LES)[16-20] in the 1990's has provided reliable threedimensional and time-dependent data to improve the investigation of wall-bounded compressible turbulent flows. Dealing with compressible effects implies to distinguish two types of density fluctuations: 1) the first one, generally associated with low Mach number flows, occurs from mean fluid-property variations (such as temperature or concentration); 2) the second one arises from the compressible nature of the fluid, at high Mach number, when significant pressure fluctuations produces density variations. Nevertheless, in the study of wall-bounded compressible turbulent flows it is well accepted that compressibility effects are mainly due to mean fluid-property variations[21]. This convenient assumption, referred to as Morkovin hypothesis, allows to investigate the compressible turbulent boundary layer in the same line of the incompressible one by paying attention to the effects of mean density variations. The use of the Van Driest transformation[22] for the modeling of compressible flows is thus supported by this hypothesis, even at high Mach number. Experimental[23-26] and numerical[15, 18, 27, 28] studies have shown the accuracy of the Van Driest transformation in scaling the velocity profile with wall heat transfer and/or high Mach number flows.

The case of reacting flows where chemistry can modify the wall turbulence structure has received little attention in the literature. Martin and Candler[29, 30] were the first, and to the authors's knowledge the only ones, who performed a DNS of hypersonic reacting boundary layer. Their studies focus on the feedback mechanism between chemistry and turbulence: exothermic reactions provide energy to the turbulent motion while the reaction rate is increased by the turbulent temperature fluctuations. As a consequence, exothermic reactions increase the magnitude of turbulent fluctuations while the effect of endothermic ones appears to be the opposite. Despite this recent work, the understanding of near-wall turbulence structure suffers from a lack of data relevant to reacting wall bounded-flows. This is emphasized by Table I that briefly summaries the major studies available in the literature.

Reynolds Averaged Navier-Stokes (RANS) and LES methods are now widely used for the development and design of engineering devices. Wall models are often preferred in industrial calculations since they allow to reduce the computation demand drastically. Note also that 
TABLE I: Non-exhaustive overview of major studies found in the literature concerning the analysis of wall turbulence structure. References are classified according to the kind of equations implicated in the analysis.

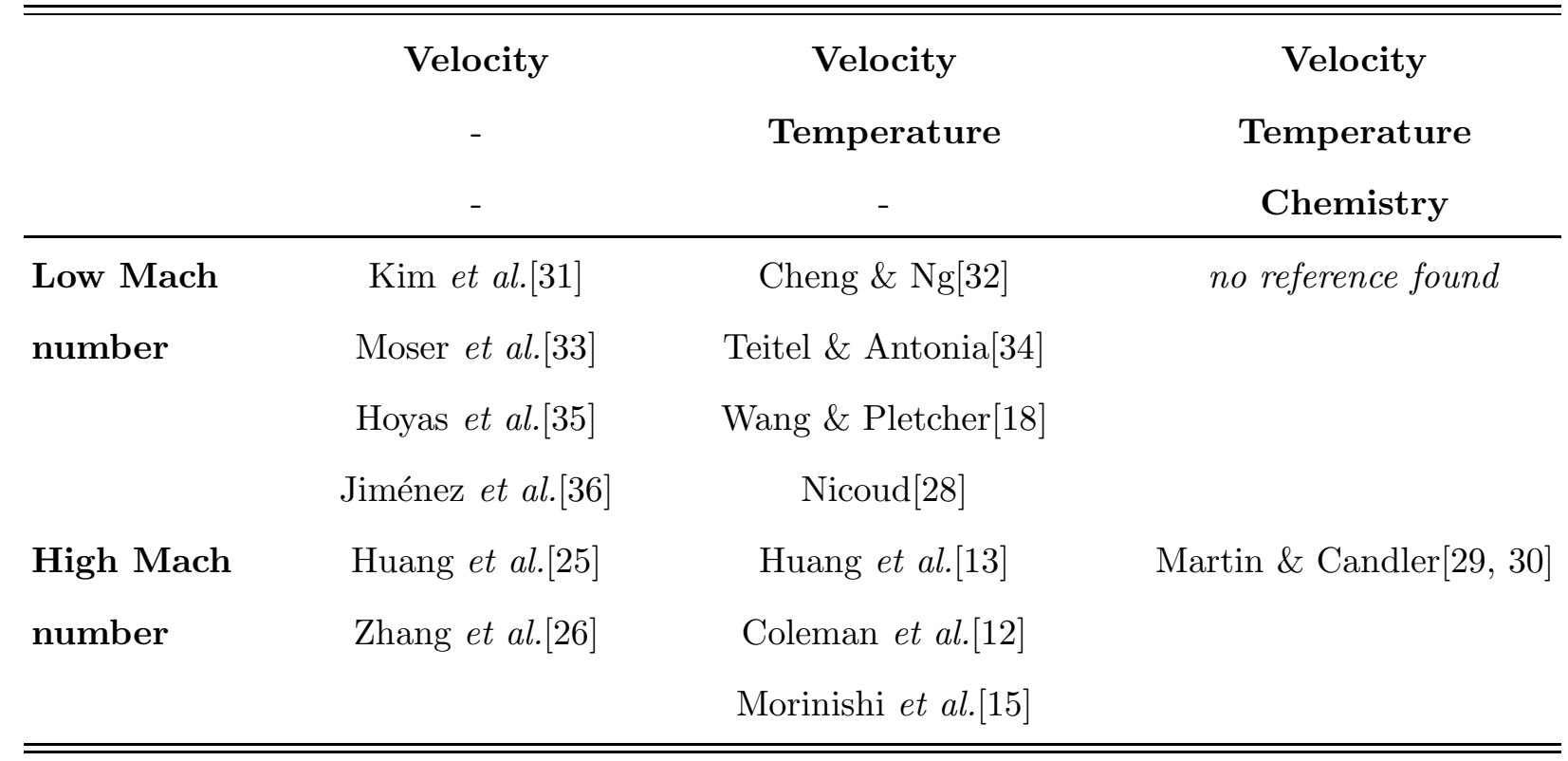

using wall models together with high-Reynolds number formulations usually leads to more stable computations than the low Reynolds number approaches where regions with small viscosity and stiff gradient must be handled[37]. The price to pay is the development of wall functions for the assessment of the mass/momentum/energy fluxes at the solid boundaries knowing the outer flow conditions at the first off-wall grid points. Note that for RANS calculations, additional prescription is necessary to insure a coherent behavior of the turbulence model transport equations in the near wall region (the specific case of $k$ - $\epsilon$ turbulence model will be discussed further, in the last section of this paper).

The logarithmic structure of the overlap region of the turbulent boundary layer is now well supported by many studies[53-56] and the classical log law is thus implemented in most of the RANS/LES codes. It provides good results for simple incompressible flows but the trend today is to generalize the wall function approach $[42,47,50,57,58]$ to account for more physics. Most of the improved models deal with streamwise pressure gradient effects[44-49], wall roughness[42, 43], compressibility[26, 39], heat transfer[41], mass transfer[38, 39], or even buoyancy effects[50], but a lack of data explains the poor advances made for reacting flows (see Table II). Moreover, generalized models often make use of artificial integration or curve-fit techniques to make the integration of equations possible[41, 50] which means 
TABLE II: Non-exhaustive list of main references found in the literature dealing with improved wall modeling. In this table, STG referred to strong temperature gradient, MNE to Mach number effect, and symbols indicate whether this type of equation is discussed $(\bullet)$ or not (-) in the corresponding reference.

\begin{tabular}{|c|c|c|c|c|}
\hline \multirow[t]{2}{*}{ Wall modeling issues } & \multirow[t]{2}{*}{ References } & \multicolumn{3}{|c|}{ Model discussed } \\
\hline & & velocity & temperature & turbulence \\
\hline Prandtl number effect & Kader $[8]$ & - & $\bullet$ & - \\
\hline \multirow[t]{2}{*}{ wall mass transfer } & Simpson $[38]$ & $\bullet$ & - & - \\
\hline & Nicoud \& Bradshaw[39] & $\bullet$ & - & - \\
\hline \multirow[t]{4}{*}{ compressibility (STG) } & Subranian \& Antonia[40] & $\bullet$ & $\bullet$ & - \\
\hline & Han \& Reitz[41] & $\bullet$ & $\bullet$ & - \\
\hline & Nicoud \& Bradshaw[39] & $\bullet$ & - & - \\
\hline & Dailey et al..[19] & $\bullet$ & $\bullet$ & - \\
\hline \multirow[t]{2}{*}{ compressibility (MNE) } & Huang \& Coleman. [27] & $\bullet$ & $\bullet$ & - \\
\hline & So et al. $[26]$ & $\bullet$ & - & - \\
\hline \multirow[t]{2}{*}{ surface roughness } & Shih et al.[42] & $\bullet$ & - & - \\
\hline & Suga et al. $[43]$ & $\bullet$ & $\bullet$ & - \\
\hline \multirow[t]{5}{*}{ streamwise pressure gradient } & Huang \& Bradshaw[44] & $\bullet$ & - & - \\
\hline & Skote \& Henningson $[45,46]$ & $\bullet$ & - & - \\
\hline & Shih et al. [47] & $\bullet$ & - & - \\
\hline & Nickels[48] & $\bullet$ & - & - \\
\hline & Houra \& Nagano[49] & $\bullet$ & $\bullet$ & - \\
\hline complex flows/mixed effects & Craft et al.[50-52] & $\bullet$ & $\bullet$ & $\bullet$ \\
\hline chemical reactions & no reference found & - & - & - \\
\hline
\end{tabular}

that they are only well suited to the applications they are derived for. It is the authors point of view that robust and accurate models can/should be developed by simplifying and integrating the basic flow equations. Such modeling effort must be supported by the analysis of detailed relevant data. Classical experimental techniques cannot provide the required space resolution for flows involving strong temperature gradients and chemical reactions. 
An alternative is to rely on DNS and wall-resolved LES to generate precise and detailed data set of generic turbulent flows under realistic operating conditions.

The purpose of the current paper is to extend the panel of the existing studies on incompressible and compressible turbulent boundary layer to the general case of wall-bounded reacting flows. The basis of our work is a set of DNS and wall-resolved LES of low Mach number periodic reacting channel flows with either small or large temperature gradients. The main objectives of the present study are:

1) generate relevant reference data in the general case of multicomponent reacting compressible turbulent boundary layer;

2) use this database to analyze momentum and energy balances;

3) illustrate how these balances can be used to improve the existing wall models and account for more physics in the wall heat flux assessment.

The paper is arranged as follows. Section II presents the governing equations of reacting flows, a short description of the numerical method, the flow parameters and the description of the simulations. Section III provides the analysis of the mass/momentum/energy balances in order to establish the quality of the results and to underline the prevalent physical mechanisms. Section IV illustrates how the database can be used to develop and improve existing wall models following a generic approach. Finally, Sec. V summarizes the results of this study.

\section{EQUATIONS AND NUMERICAL STRATEGY}

\section{A. Governing equations}

The conservation equations for three-dimensional, compressible, turbulent flows of reacting gaseous mixture are well-known and available in several text books[59, 60]. Continuity equation (1), mass species conservation (2), momentum conservation (3), total non-chemical energy conservation (4) and perfect gas equation of state (5) can be written with Einstein notation (summation on $i, j$ and $l$ subscripted variables) in the following form:

$$
\frac{\partial \rho}{\partial t}+\frac{\partial\left(\rho u_{i}\right)}{\partial x_{i}}=0
$$




$$
\begin{gathered}
\frac{\partial\left(\rho Y_{k}\right)}{\partial t}+\frac{\partial}{\partial x_{i}}\left(\rho\left(u_{i}+V_{k, i}\right) Y_{k}\right)=\dot{\omega}_{k} \\
\frac{\partial\left(\rho u_{i}\right)}{\partial t}+\frac{\partial\left(\rho u_{i} u_{j}\right)}{\partial x_{j}}=-\frac{\partial p}{\partial x_{i}}+\frac{\partial \tau_{i j}}{\partial x_{j}}+\mathcal{S}_{i} \\
\rho \frac{D E}{D t}=-\frac{\partial q_{i}^{*}}{\partial x_{i}}+\frac{\partial}{\partial x_{j}}\left(\tau_{i j} u_{i}\right)-\frac{\partial}{\partial x_{i}}\left(p u_{i}\right)+\dot{\omega}_{T}+\mathcal{Q}+u_{i} \mathcal{S}_{i} \\
\frac{p}{\rho}=r T
\end{gathered}
$$

with $\tau_{i j}$ the viscous shear stress tensor and $q_{i}$ the molecular heat flux (different from $q_{i}^{*}$ that represents the sensible enthalpy flux) respectively given by:

$$
\begin{gathered}
\tau_{i j}=-\frac{2}{3} \mu \frac{\partial u_{l}}{\partial x_{l}} \delta_{i j}+\mu\left(\frac{\partial u_{i}}{\partial x_{j}}+\frac{\partial u_{j}}{\partial x_{i}}\right) \\
q_{i}=\underbrace{-\lambda \frac{\partial T}{\partial x_{i}}+\rho \sum_{k} h_{s, k} Y_{k} V_{k, i}}_{q_{i}^{*}}+\rho \sum_{k} \Delta h_{f, k}^{0} Y_{k} V_{k, i}
\end{gathered}
$$

The notations used in this paper are the following: $i$ subscripted quantities refer to variables dependent on directions $\vec{x}, \vec{y}$ and $\vec{z}$ (for multiple summation, subscript $i$ is replaced by either $j$ or $l$ ); subscript $k$ refers to $k^{t h}$ species; for any quantity $f, D f / D t=\partial f / \partial t+$ $u_{i} \partial f / \partial x_{i} ; u_{i}$ is the velocity vector whose components are noted $u, v$ and $w$ in the cartesian coordinate system; $p$ is the thermodynamic pressure; $T$ the temperature; $\rho$ the density of the fluid; $Y_{k}$ and $X_{k}$ denotes the mass and molar fractions of species $k$ respectively, with $Y_{k}=X_{k} W_{k} / W ; W_{k}$ the atomic weight of species $k ; W=\sum_{k} X_{k} W_{k}$ the mean molecular weight of the mixture; $r=R / W$ the perfect gas constant per mass unit with $R$ the perfect gas constant per mole; $V_{k, i}$ the diffusion velocity of species $k$ in $i$-direction; $\dot{\omega}_{k}$ the mass reaction rate of species $k ; \dot{\omega}_{T}=-\sum_{k} \Delta h_{f, k}^{0} \dot{\omega}_{k}$ is the heat release with $\Delta h_{f, k}^{0}$ the chemical enthalpy of formation per unit mass of species $k$ (note that when one considers the conservation equation for temperature instead of Eq. (4), the reaction source term not only involves the formation enthalpy but also the sensible one; this could be confusing since this term is also called "heat release" in the literature whereas its definition is different[60]); $\mathcal{S}_{i}$ the momentum source term acting in the $i$-direction and $\mathcal{Q}$ the energy volume source term (see Sec. II C where the values 
retained for these source terms are specified); $C_{p, k}$ the heat capacity at constant pressure of species $k ; C_{p}=\sum_{k} C_{p, k} Y_{k}$ and $C_{v}$ the heat capacity at constant pressure and constant volume of the mixture, respectively; $\mu$ and $\nu=\mu / \rho$ are the dynamic and the kinematic viscosity, respectively; $\lambda$ the heat diffusion coefficient of the fluid; the possible variables to represent enthalpy for one species are the sensible enthalpy $h_{s, k}=\int_{T_{0}}^{T} C_{p, k} d T$ or the specific enthalpy, $h_{k}=\int_{T_{0}}^{T} C_{p, k} d T+\Delta h_{f, k}^{0}$ (sum of the sensible and the chemical parts), defined with a reference temperature $T_{0}=0 K$ for this work; the possible variables to represent the enthalpy of the mixture are the sensible enthalpy $h_{s}=\sum_{k} h_{s, k} Y_{k}$, the specific enthalpy $h=h_{s}+\sum_{k} \Delta h_{f, k}^{0} Y_{k}$, the total enthalpy $h_{t}=h+u_{i} u_{i} / 2$ or the total non chemical enthalpy $H=h_{s}+u_{i} u_{i} / 2$; by analogy, $e_{s}, e, e_{t}$ and $E$ denote the sensible, specific, total and total non chemical energies, respectively (one recalls that $p / \rho$ is the difference between enthalpy and energy, whatever the form retained is).

The derivation of the system of equations (1-7) is performed under the following assumptions:

- no external forces,

- effects of volume viscosity are null,

- no Dufour effect for the heat flux,

- radiation heat transfer is negligible.

The latter statement seems questionable regarding the high temperatures and strong temperature variations involved in this study (see Table IV). However, the study of Amaya et al.[61] has demonstrated that the changes introduced by the radiative source term in such configurations do not have an incidence upon the turbulence structure of the flow. This means that taking into account the radiative effects or not will lead to the same wall model development. Besides, it appears that for a periodic turbulent channel flow configuration such as case B of the present work (see Table IV and Table V), neglecting radiative heat transfer only fathers a relative error of $7 \%$ in the prediction of the total wall heat flux.

Moreover, even if the density can vary in the computation, the buoyancy effects are neglected for two main reasons. First, it is often necessary to separate different physical effects in order to improve understanding of their fundamentals. Neglecting the buoyancy effects allows us to focus on the proper density effects. Second, an estimation of the ratio 
$G r / \operatorname{Re}^{2}$ ( $G r$ being the Grashof number) sustains this assumption. Indeed, with a moderate Reynolds number $\left(R e=5000\right.$ ), a relative density ratio $\Delta \rho / \rho_{w}=0.67$ (maximum value taken in the wall-resolved LES), and a kinematic viscosity of order $\nu \approx 10^{-5} \mathrm{~m}^{2} / \mathrm{s}$, we find that $G r / R e^{2} \approx 2630 h^{3}$ ( $h$ is the reference lenght, e.g. the channel half-height in this study). Thus, the parameter $G r / R e^{2}$ is smaller than $5 \%$ (much less than the value 0.3 advocated by Sparrow et al.[62] for the critical limit between forced convection and mixed flows) as long as $h$ is smaller than 0.026 meters. This means that the no-buoyancy body force assumption is justified whether the characteristic length scale is of order $2 \mathrm{~cm}$ or less. This would be a reasonable range for a true experiment, for instance based on a subscale solid rocket motor designed to measure heat fluxes[63]. For the present simulations, this condition is also verified as the channel half-height never exceeds $0.2 \mathrm{~mm}$.

\section{Note concerning the approximation of perfect gas equation of state}

Because the mean temperature and pressure of the mixture are very high in this study (around $3000 \mathrm{~K}$ and $10 \mathrm{MPa}$, respectively) the validity of the perfect gas equation of state, Eq.(5), is also questionable. Indeed, if these operating conditions are close to the critical point or near the pseudo-boiling line, the classical perfect gas equation of state should be modified to take into account attractive and repulsive intermolecular forces[64]. Hence, in order to support the usability of Eq.(5), the Peng-Robinson equation of sate[65] has been used to compute the compressibility factor, $Z=P \mathcal{V} / R T$ where $\mathcal{V}$ is the molar volume of the mixture. As presented in Table III, $Z$ stays very close to unity for the three tested temperatures. This indicates that replacing Eq.(5) by a real gas equation of state (such as the Peng-Robinson one[65]) should not have any major repercussions on the thermodynamics of the flow. Moreover, the goal of this study is to develop a wall model that gives reliable predictions in the most common cases (the perfect gas assumption is valid for $95 \%$ of industrial applications); the use of a real gas equation of state is thus out of the scope of this work. 
TABLE III: Compressibility factor of the simulated mixture computed with the Peng-Robinson equation of state. Results obtained for three characteristic temperatures of the present study.

\begin{tabular}{cc}
\hline \hline Temperature $(K)$ & Compressibilityfactor \\
\hline $1050 K$ & 1.02 \\
$2750 K$ & 1.009 \\
$3000 K$ & 1.008 \\
\hline \hline
\end{tabular}

\section{B. Modeling of the transport terms}

When external forces acting on the species are neglected, the exact expression of the diffusion velocity is reduced to:

$$
V_{k, i}=\underbrace{-\sum_{l} D_{k l} \frac{\partial X_{l}}{\partial x_{i}}}_{\text {mixture effect }} \underbrace{-\sum_{l} D_{k l}\left(X_{l}-Y_{l}\right) \frac{1}{p} \frac{\partial p}{\partial x_{i}}}_{\text {pressure gradient effect }} \underbrace{-\sum_{l} D_{k l} \chi_{l} \frac{\partial \ln T}{\partial x_{i}}}_{\text {Soret effect }}
$$

where $D_{k l}$ are the multicomponent diffusion coefficients of the diffusion matrix and $\chi_{l}$ the thermal diffusion ratio of species $l$. Solving the transport system Eq. (8) is an expensive task for CFD codes and this is why the system is simplified in the present study. First of all, the pressure gradient effect is neglected because for a periodic channel flow configuration the pressure variations stay weak. The Soret effect is also neglected and the simplification of Eq. (8) is achieved by the use of the Hirschfelder and Curtiss approximation[66] with correction velocity. As mentioned by Giovangigli[67] this is the best first-order accuracy model for estimating diffusion velocities of a multicomponent mixture. It consists in replacing the rigorous mixture effect part of the diffusion velocity system by a simpler one:

$$
V_{k, i}^{h c} X_{k}=-\mathcal{D}_{k} \frac{\partial X_{k}}{\partial x_{i}}
$$

where the $V_{k, i}^{h c}$ denotes the Hirschfelder and Curtiss diffusion velocity, and $\mathcal{D}_{k}$ an equivalent diffusion coefficient of species $k$ into the rest of the mixture. The latter coefficient is built from the binary diffusion coefficients $\mathcal{D}_{i j}$ which can be assessed from the gas kinetic theory $[67]$ :

$$
\mathcal{D}_{k}=\frac{1-Y_{k}}{\sum_{j \neq k} X_{j} / \mathcal{D}_{j k}}
$$

Mass conservation is a specific issue when dealing with reacting flows. To insure that the system of equations satisfies the two constraints $\sum_{k} Y_{k}=1$ and $\sum_{k} Y_{k} V_{k, i}=0$, a correction 
velocity $V_{i}^{\text {cor }}$ is added to the Hirschfelder and Curtiss diffusion velocity $V_{k, i}^{h c}$. At each time step, the correction velocity is computed as:

$$
V_{i}^{c o r}=\sum_{k} \mathcal{D}_{k} \frac{W_{k}}{W} \frac{\partial X_{k}}{\partial x_{i}}
$$

so that the diffusion velocities for each species $k$ :

$$
V_{k, i}=V_{k, i}^{h c}+V_{i}^{c o r}
$$

satisfy the constrain $\sum_{k} Y_{k} V_{k, i}=0$. Combined with the assumption of constant Schmidt numbers, the Hirschfelder and Curtiss approximation is very convenient because the equivalent diffusion coefficients can be easily related to the kinematic viscosity according to: $\mathcal{D}_{k}=\nu / S c_{k}$. The problem is then efficiently closed by imposing the Schmidt numbers and it is not necessary to compute the $\mathcal{D}_{i j}$ coefficients which are complex functions of collision integrals and thermodynamics variables. Note that in the present work, the dynamic viscosity is modeled by a power-law expression:

$$
\mu=\mu_{\text {ref }}\left(\frac{T}{T_{r e f}}\right)^{c}
$$

where $\mu_{\text {ref }}=8.194 \times 10^{-5}$ Pa.s, $T_{\text {ref }}=3000 \mathrm{~K}$ and $c=0.656$. This choice is supported by Fig. 1 that presents the difference between the retained model and the dynamic viscosity of the simulated mixture computed with EGLIB library[68, 69]. This plot argues that a Sutherland type formulation is not necessary for the temperature range covered in this study.

\section{Set up of the simulations}

In order to cover different kinds of turbulent wall-bounded flows and to isolate some physical processes such as chemical reactions or strong temperature gradients, four different periodic channel flows have been computed. Their physical characteristics are summarized in Table IV in which $R e_{\tau}$ is the target friction Reynolds number, $R e_{c}$ the Reynolds number based on the channel half-height (denoted by $h$ in this paper) and centerline properties, and $R e_{b}$ the Reynolds number based on bulk quantities (i.e. the bulk density $\rho_{b}=1 / h \int_{0}^{h} \bar{\rho} d y$, bulk velocity $u_{b}=1 / h \int_{0}^{h} \overline{\rho u} d y / \rho_{b}$, and viscosity at the wall). Note that case $\mathrm{A}$ is the reference case for this study because this is the one that includes less physical effects: the temperature gradient is small and no chemical reaction is activated. 


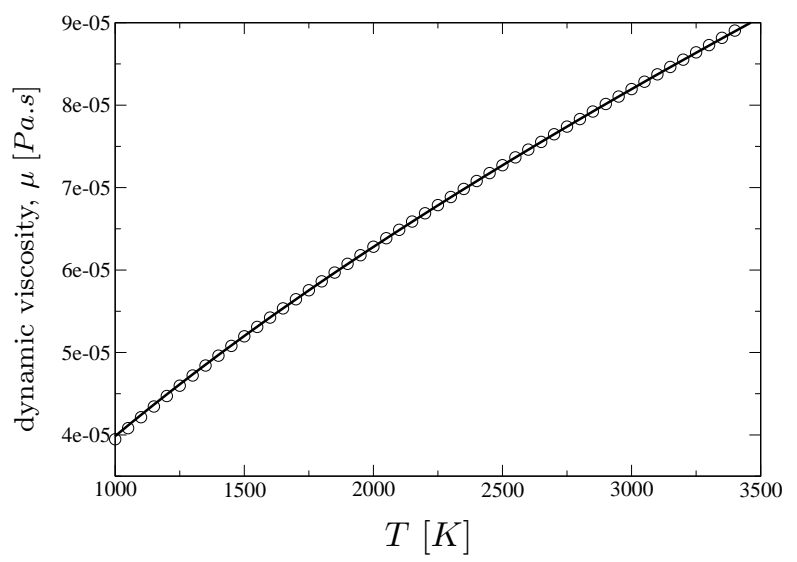

FIG. 1: Dynamic viscosity of the simulated mixture as a function of temperature. $\quad$ : computed with EGLIB library; — : power-law Eq. (13).

Figure 2 shows the reference coordinate system, and the notation used to describe the computational domain. Periodic boundary conditions are applied in the homogeneous streamwise $(\vec{x})$ and spanwise $(\vec{z})$ directions while the temperature at the walls is imposed by an isothermal boundary condition. The pressure gradient that drives the flow and compensates for viscous dissipation is enforced by adding a space and time constant source term in the streamwise direction. Hence, the momentum source term $\mathcal{S}_{i}$ of Eq. (3) and Eq. (4) is null in the wall normal and spanwise directions but takes a constant value, noted $\mathcal{S}_{x}$, in the streamwise direction.

In addition, a source term, noted $\mathcal{Q}$, that warms the fluid in volume is added to the energy equation (4) in order to drive the mean temperature of the wall-bounded flow to the desired value $T_{\text {mean }}$. At each time step, a space constant value of $\mathcal{Q}$ is adjusted dynamically according to:

$$
\mathcal{Q}^{t+1}=\rho_{a v} C_{v, a v} \frac{T_{\text {mean }}-\frac{1}{V} \iiint_{\Omega} T^{t} d V}{\tau_{\text {relax }}}
$$

where subscript "av" denotes an averaged quantity integrated over space and time variables, $\mathcal{Q}^{t+1}$ the energy source term at time $t+\Delta t, \Omega$ the computational domain and $V$ its volume, $T^{t}$ the temperature at time $t$, and $\tau_{\text {relax }}$ a relaxation time coefficient set in the present study to $\tau_{\text {relax }}=0.3 h / u_{\tau}$.

\section{Domain size and spatial resolution}

The original Kim, Moin and Moser[31] channel calculation was at low Reynolds number, 


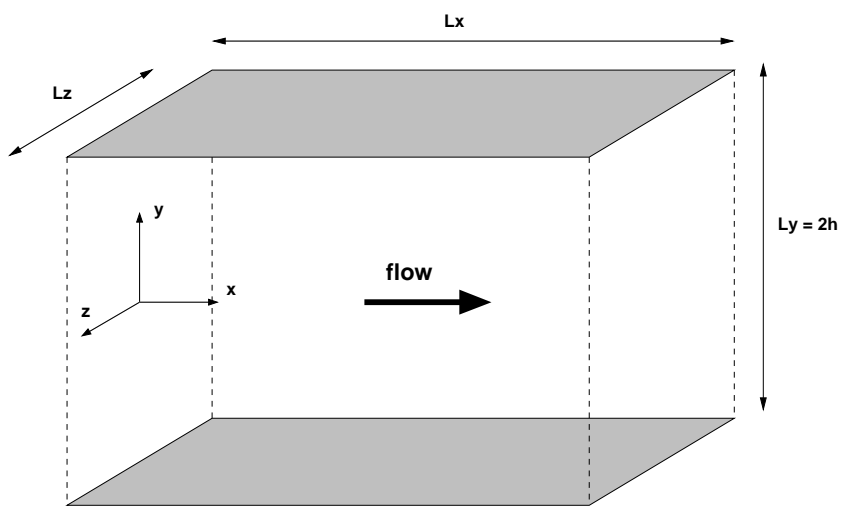

FIG. 2: Sketch of a computational domain.

TABLE IV: Physical parameters of the simulated cases. In this table, $T_{w}$ and $T_{c}$ are the temperature respectively at the wall and at the centerline, and the Mach number is the maximum value evaluated with local properties.

\begin{tabular}{cccccccc}
\hline \hline case & $R e_{\tau}$ & $R e_{c}$ & $R e_{b}$ & Mach & $T_{w}$ & $T_{c} / T_{w}$ & chemistry \\
\hline A & 300 & 4400 & 4130 & 0.2 & $2750 K$ & 1.1 & off \\
B & 300 & 4460 & 4160 & 0.2 & $2750 K$ & 1.1 & activated \\
C & 1000 & 5080 & 10090 & 0.2 & $1050 K$ & 3 & off \\
D & 1000 & 5190 & 10150 & 0.2 & $1050 K$ & 3 & activated \\
\hline \hline
\end{tabular}

$R e_{\tau}=180$. One recalls that $R e_{\tau}=h u_{\tau} / \nu_{w}, h$ being the channel half-height, $u_{\tau}=\sqrt{\overline{\tau_{w}} / \overline{\rho_{w}}}$ the friction velocity and $\nu_{w}$ the kinematic viscosity at the wall. Several studies[33, 35] have later shown that it was necessary to simulate channel flows in wider boxes to account for turbulent structures of high Reynolds number and to expect a sufficiently large log layer. Hence, a minimal target value of $R e_{\tau}=300$ has been used in the present work. Other parameters relevant to the numerical resolution are summarized in Table V. For each DNS case, this table presents: the type of the simulation; the number of grid points $n_{x} \times n_{y} \times n_{z}$ (all the simulations were carried out with hexahedral elements); the streamwise and spanwise length of the domain scaled by the channel half-height; $\Delta x^{+}$and $\Delta z^{+}$, the $x$ and $z$ constant grid spacing scaled by the viscous length $y_{\tau}=\nu_{w} / u_{\tau} ; \Delta y_{w}^{+}$and $\Delta y_{c}^{+}$, the minimum and maximum grid spacing in viscous wall units, respectively at the wall and at the center of the channel (the grid is stretched in the wall normal direction); the computational time period $t$ scaled by the characteristic diffusion time, $\tau_{\text {diff }}=h / u_{\tau}$. 
TABLE V: Numerical parameters of the simulations.

\begin{tabular}{cccccccccc}
\hline \hline case & type of simulation & $n_{x} \times n_{y} \times n_{z}$ & $L_{x} / h$ & $L_{z} / h$ & $\Delta x^{+}$ & $\Delta z^{+}$ & $\Delta y_{w}^{+}$ & $\Delta y_{c}^{+}$ & $t / \tau_{\text {diff }}$ \\
\hline A & DNS & $31 \times 159 \times 39$ & 3.14 & 1.25 & 29 & 9 & 0.8 & 6 & 25 \\
B & DNS & $31 \times 159 \times 39$ & 3.14 & 1.25 & 29 & 9 & 0.8 & 6 & 25 \\
C & \multirow{2}{*}{ wall-resolved LES } & $44 \times 159 \times 44$ & 3.5 & 1.3 & 81 & 30 & 1.4 & 30 & 14 \\
D & \multirow{2}{*}{ wall-resolved LES } & $44 \times 159 \times 44$ & 3.5 & 1.3 & 81 & 30 & 1.4 & 30 & 14 \\
\hline \hline
\end{tabular}

The values of the length of the computational box and the number of grid points seem to be very small compared to the recent simulation of Hoyas an Jiménez[35]. However, the goal here is not to provide the "exact" high-order turbulence statistics but rather to represent zeroth order effects of the density variation and chemistry on the turbulent profiles: concerning the box size, the domain has been built to be about twice wider than the minimal unit flow conditions defined by Jiménez and Moin[70]; concerning the grid spacing, a particular attention has been given to the wall normal refinement (first off-wall grid point situated at $y^{+} \approx 1$ ) and the constant streamwise and spanwise spacing has been set to a typical value.

An indication of the numerical fidelity of the calculations is given by the streamwise and spanwise two-point correlations from the channel centerline and near the walls, shown in Fig. 3 for case A. These results are similar to those found from cases B, C and D. The correlation coefficients should drop to zero if the computational domain were large enough to allow the use of periodic conditions in the homogeneous directions. Except for the pressure two-point correlation near the centerline region, the domain size appears to be wide enough. In fact, the pressure stay correlated because of the acoustic waves that can travel along the "infinitely" long channel without being damped. Note however that the two-point correlation of the streamwise velocity goes to zero as expected, meaning that the acoustic fluctuations are not large enough to modify the turbulent statistics. This behavior has also been reported by Coleman et al.[12].

Moreover, the time convergence of the statistics has been verified looking at the time evolution of the root-mean-square streamwise velocity profile, a first-order statistics. This result is shown in Fig. 4 for case D, case for which the assimilation time period is the shortest (the time convergence of the statistics is less questionable for cases $\mathrm{A}$ and $\mathrm{B}$ because their assimilation period is longer). This plot demonstrates the proper convergence 

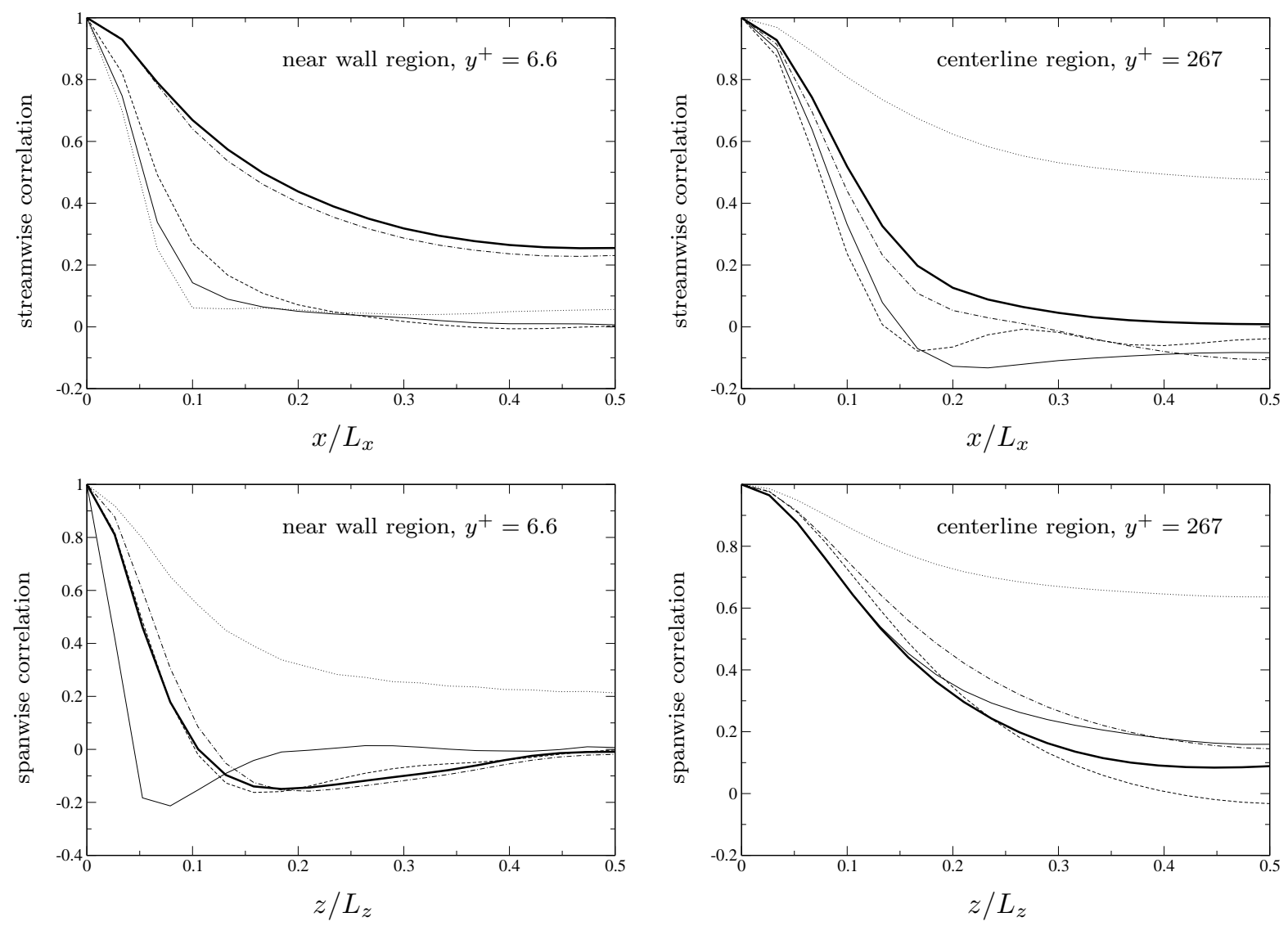

FIG. 3: Two-point correlations in streamwise and spanwise directions for case A. $\mathrm{u}$; : v; ----: w; - - : T; $\quad \cdots \cdots \cdot$ : p.

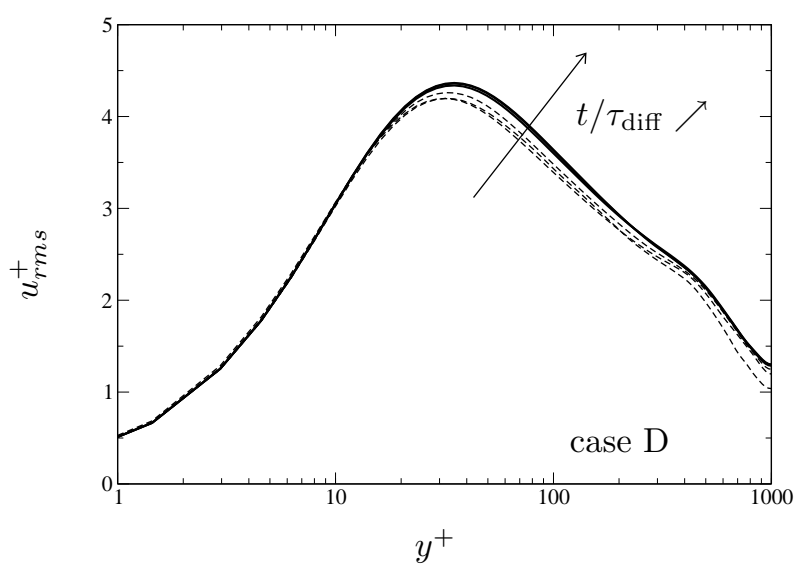

FIG. 4: Time convergence of the root-mean-square streamwise velocity in wall units (results are similar for case C). $\quad----: t / \tau_{\text {diff }}<10$ (three profiles are plotted namely, $t / \tau_{\text {diff }}=1,5,8$ ); $: t / \tau_{\text {diff }}>10$ (four profiles are plotted namely, $t / \tau_{\text {diff }}=11,12,13,14$ ). 
TABLE VI: Transport coefficients of the simulated mixture.

\begin{tabular}{cccccccc}
\hline \hline $\operatorname{Pr}$ & $S c, \mathrm{H}_{2}$ & $S c, \mathrm{H}$ & $S c, \mathrm{H}_{2} \mathrm{O}$ & $S c, \mathrm{OH}$ & $S c, \mathrm{CO}_{2}$ & $S c, \mathrm{CO}$ & $S c,,_{2}$ \\
\hline 0.47 & 0.2 & 0.15 & 0.65 & 0.54 & 0.98 & 0.86 & 0.87 \\
\hline \hline
\end{tabular}

of the statistics for $t / \tau_{\text {diff }}>10$.

\section{Simulated mixture}

The mixture retained for the simulations is composed of seven species: $\mathrm{H}_{2}, \mathrm{H}, \mathrm{H}_{2} \mathrm{O}$, $\mathrm{OH}, \mathrm{CO}_{2}, \mathrm{CO}$ and $\mathrm{N}_{2}$. These species have been selected because they are typical of many industrial applications and they offer a large range of molar weight and thermo-physical properties, thus promoting the multispecies effects. Usual values of transport properties, such as Schmidt and Prandtl numbers, are determined using the EGLIB library[68, 69] and summarized in Table VI. Note that in this study, the Prandtl number of the mixture is equal to 0.47 which differs from the classical value 0.7 for air. Hence, the classical wall models for temperature specially developed for air flow configuration will not be efficient in the present DNS. For this reason, and because the main objective is to build a reliable model which would not be limited to a unique Prandtl number value, it will be shown in Sec. IV how the Prandtl number dependance can be included into the model.

Moreover, this equivalent mixture needs a reliable kinetic scheme which reproduces the concentration changes of each species during the simulations. Hence, in the framework of Maxwellian production rates[67], $\dot{\omega}_{k}$ the mass reaction rate of species $k$ produced by $R$ reactions is written:

$$
\dot{\omega}_{k}=W_{k} \sum_{r=1}^{R}\left(\nu_{k, r}^{b}-\nu_{k, r}^{f}\right) Q_{r}
$$

where $\nu_{k, r}^{f}$ and $\nu_{k, r}^{b}$ are the forward and backward stoichiometric coefficients of the species $k$ in reaction $r$, respectively, and $Q_{r}$ the molar progress rate of reaction $r$. For the general case, this rate of progress $Q_{r}$ is usually given by:

$$
Q_{r}=\gamma_{M}\left(K_{r}^{f} \prod_{k} \gamma_{k}^{\nu_{k, r}^{f}}-K_{r}^{b} \prod_{k} \gamma_{k}^{\nu_{k, r}^{b}}\right)
$$

where $\gamma_{k}=\rho Y_{k} / W_{k}$ is the molar concentration of species $k$. For three-body reactions $\gamma_{M}=\sum_{k} \alpha_{k, r} \gamma_{k}$ is the molar concentration of the equivalent third body $M$ with $\alpha_{k, r}$ the 
TABLE VII: Chemical kinetic scheme retained for this study. The first and fifth reactions involve an abstract third body, denoted by $M$. For these reactions the third-body efficiencies are set by default to $\alpha_{k, r}=1.0$ for all the species, except for the ones given in the table.

\begin{tabular}{rccccc}
\hline \hline Reaction & $A[\mathrm{cgs}$ units $]$ & $\beta$ & $E_{a}[\mathrm{cal} / \mathrm{mole}]$ & $\alpha_{k, r}$ \\
\hline $2 \mathrm{H}+\mathrm{M} \rightleftharpoons \mathrm{H}_{2}+M$ & $1.00 \mathrm{E}+18$ & -1.0 & 0. & $\alpha_{H_{2}}=\alpha_{\mathrm{H}_{2} O}=0.0$ \\
$2 \mathrm{H}+\mathrm{H}_{2} \rightleftharpoons 2 H_{2}$ & $9.00 \mathrm{E}+16$ & -0.6 & 0. & - \\
$2 \mathrm{H}+\mathrm{H}_{2} \mathrm{O}$ & $\rightleftharpoons H_{2}+\mathrm{H}_{2} \mathrm{O}$ & $6.00 \mathrm{E}+19$ & -1.25 & 0. & - \\
$2 \mathrm{H}+\mathrm{CO}_{2}$ & $\rightleftharpoons \mathrm{H}_{2}+\mathrm{CO}_{2}$ & $5.50 \mathrm{E}+20$ & -2.0 & 0. & - \\
$\mathrm{H}+\mathrm{OH}+\mathrm{M}$ & $\rightleftharpoons \mathrm{H}_{2} \mathrm{O}+\mathrm{M}$ & $2.20 \mathrm{E}+22$ & -2.0 & 0. & $\alpha_{H_{2}}=0.73 ; \alpha_{H_{2} O}=3.65$ \\
$\mathrm{OH}+\mathrm{H}_{2} \rightleftharpoons \mathrm{H}+\mathrm{H}_{2} \mathrm{O}$ & $2.16 \mathrm{E}+08$ & 1.51 & 3430. & - \\
$\mathrm{OH}+\mathrm{CO}$ & $\rightleftharpoons \mathrm{H}+\mathrm{CO}_{2}$ & $4.76 \mathrm{E}+07$ & 1.228 & 70. & - \\
\hline \hline
\end{tabular}

third-body efficiency of species $k$ in reaction $r$ (when no third body is involved in the reaction $\gamma_{M}=1$ by default). $K_{r}^{f}$ and $K_{r}^{b}$ denotes the forward and backward rate constants of reaction $r$, respectively. In this study, the forward rate constants are approximated by an Arrhenius formulation:

$$
K_{r}^{f}=A T^{\beta} \exp \left(-\frac{E_{a}}{R T}\right)
$$

where $A$ is the pre-exponential constant, $\beta$ the temperature exponent, and $E_{a}$ the activation energy. The backward rate constants are evaluated through the equilibrium constants, $K_{r}^{e}=K_{r}^{f} / K_{r}^{b}$, which could be assessed from classical theory[59]. Thereby, a kinetic scheme based on seven chemical reactions has been tuned using the GRI-Mech elementary equations (URL: http://www.me.berkeley.edu/gri_mech). Details of the retained scheme are presented in Table VII. It has been validated verifying that this reduced scheme implemented in the code is able to predict the right chemical equilibrium composition given by the EQUIL library of CHEMKIN (software developed at Sandia National Laboratories for solving complex chemical kinetics problems) and the whole set of species involved in GRI-Mech chemical reaction mechanism. 


\section{Numerical solver}

DNS/LES were performed with the AVBP solver developed at CERFACS. This parallel code offers the capability to handle unstructured or structured grids in order to solve the full 3D compressible reacting Navier-Stokes equations with a cell-vertex formulation. During the past years, its efficiency and accuracy have been widely demonstrated in both LES and DNS for different flow configurations[71-73].

A centered Galerkin finite element method with a three-step Runge-Kutta temporal integration method has been used in the present study to solve the flow equations described above. This numerical scheme is fourth order accurate in space, third order accurate in time, and thus compatible with the grid resolution used (see Table V) to provide reliable statistics of the flow. The efficiency of this numerical method has been successfully tested by Colin \& Rudgyard[74].

Finally, the subgrid-scale stress model applied for wall-resolved LES is the WALE (WallAdapting Local Eddy-viscosity) model[75] specially developed for this kind of wall-bounded flows. It is notably able to recover the proper $y^{3}$ damping scaling for eddy viscosity at the wall.

\section{DNS RESULTS}

The forthcoming sections present the profiles obtained after a statistical treatment has been applied to the simulation results. It consists in performing averages over the homogeneous directions $(\vec{x}$ and $\vec{z}$ ) and time (the integration time is given in Table $\mathrm{V}$ ). This averaging process implies that any partial derivative of a variable $f$ in the wall normal direction is equivalent to a total derivative, i.e. $\partial f / \partial y \equiv d f / d y$. Let us introduce the notations used in the equations that follow: either $\bar{*}$ or $\langle\cdot\rangle$ for the ensemble average; either $\widetilde{\cdot}$ or $\{\cdot\}$ for the Favre average defined for a variable $f$ as $\{f\}=\langle\rho f\rangle /\langle\rho\rangle$; the single prime, ', and the double prime, ", represent the turbulent fluctuations with respect to Reynolds and Favre averages respectively.

A wall model allows to predict wall quantities such as the shear stress, $\tau_{w}$, and the heat flux, $q_{w}$, from the knowledge of the flow quantities far from the wall. Typically, these two parameters are functions of velocity and temperature but in the context of multicomponent 

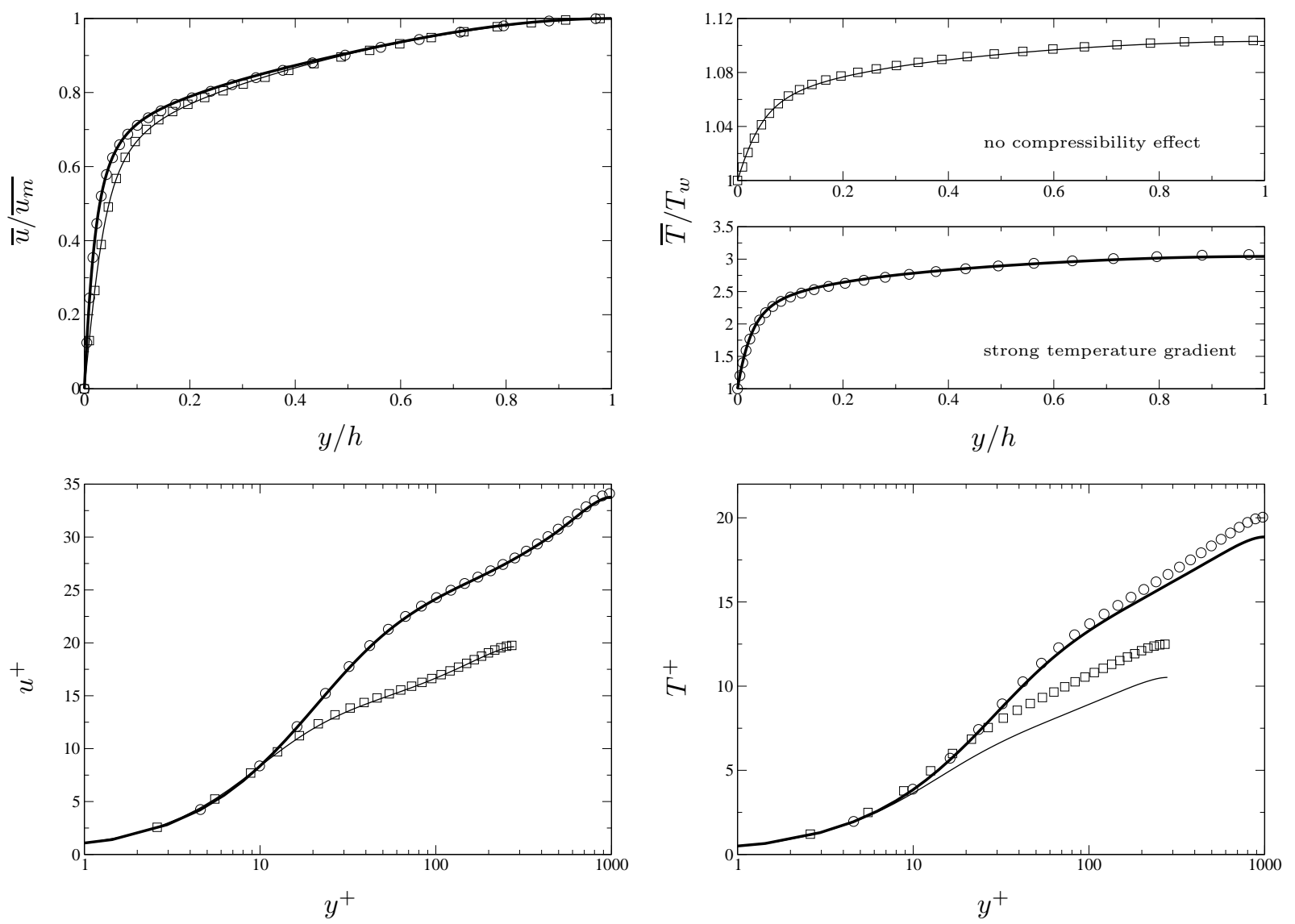

FIG. 5: Effect of chemical reactions and strong temperature gradient on mean velocity and temperature profiles. $\quad \square$ : case A (no chemistry); — $\quad$ : case B; $\mathrm{O}$ : case C (no chemistry); - : case D.

reacting flows, the mixture composition dependence has to be included in wall models. This is emphasizes by Fig. 5 that depicts the differences induced by chemical reactions and a significant temperature gradient on mean velocity and temperature profiles. On the top row of this figure, the velocity is scaled by the maximum velocity at the centerline, $\overline{u_{m}}$, and the mean temperature by the temperature at the wall, $T_{w}$ (note that because the present study makes use of isothermal walls, one has $T_{w} \equiv \overline{T_{w}}$ as well as $\mu_{w} \equiv \overline{\mu_{w}}$; this equivalence does not stand for other variables at the wall). On the bottom row, profiles are shown in the standard wall unit scaling: $u^{+}=\bar{u} / u_{\tau}$ and $y^{+}=y u_{\tau} / \overline{\nu_{w}}$ with $u_{\tau}=\sqrt{\overline{\tau_{w}} / \overline{\rho_{w}}}$, and $T^{+}=\left(T_{w}-\bar{T}\right) / T_{\tau}$ with $T_{\tau}=\overline{q_{w}} /\left(\overline{\rho_{w}} \overline{C_{p, w}} u_{\tau}\right)$. This figure clearly shows the coupling existing between velocity, temperature, and chemistry. Concerning $u^{+}$profiles, chemistry has a negligible influence and the only visible discrepancies are due to density variations (related to strong temperature 
gradient). Concerning $T^{+}$profiles (representing the temperature profile scaled by the wall heat flux), the discrepancies observed for the four simulated cases indicate that the wall heat flux is both sensitive to chemistry and strong temperature gradient, and that none of these two effects can be neglected compared to the other one. In others words, even if chemical reactions do not seem to have an influence on the mean velocity and temperatures profiles (top row of Fig. 5), they actually have an important effect on the fluxes at the wall. This justifies the need of taking care of fluid heterogeneity in wall models. Hence, the coupled wall functions derived should have the form of a system of two equations $\tau_{w}=\tau_{w}\left(u, T, Y_{k}\right)$ and $q_{w}=q_{w}\left(u, T, Y_{k}\right)$.

First of all, the quality of the simulations and the efficiency of the averaging procedure will be tested by verifying that the balance of each conservation equation is well closed. The study of species mass fraction/momentum/energy balances will then help in understanding the behavior of reacting compressible turbulent wall-bounded flows, investigating which terms could be neglected in the turbulent momentum/energy transfers for developing a new wall model. For clarity reasons and because this study mostly focusses on multicomponent/chemistry effects, results from cases B and D will be mainly shown in what follows.

\section{A. Species mass fraction balances}

The averaging procedure for equation (2) leads to the following expression:

$$
\frac{d\left(\bar{\rho} \widetilde{v Y_{k}}\right)}{d y}+\frac{d\left(\bar{\rho} \widetilde{V_{k, y} Y_{k}}\right)}{d y}=\overline{\dot{\omega}_{k}}
$$

where the first left hand side (LHS) term can be assimilated to the divergence of the convective velocity field associated to species $k$, the second one to the divergence of the diffusion velocity field for the $k^{\text {th }}$ species and the right hand side (RHS) term to the mean production rate of species $k$. Figure 6 compares the influence of LHS and RHS terms of Eq. (18) for each species. The balances are well closed which indicates that the simulation is sufficiently time-converged and that the averaging time is long enough to proceed to an analysis of these balances (the maximum closure error is around $5 \%$ at the wall for species $O H$ ). Another good criterion to validate the quality of the DNS and of its post-processing is to check that the condition $\widetilde{v}=0$, resulting from the continuity equation (1) and the no-slip condition at 

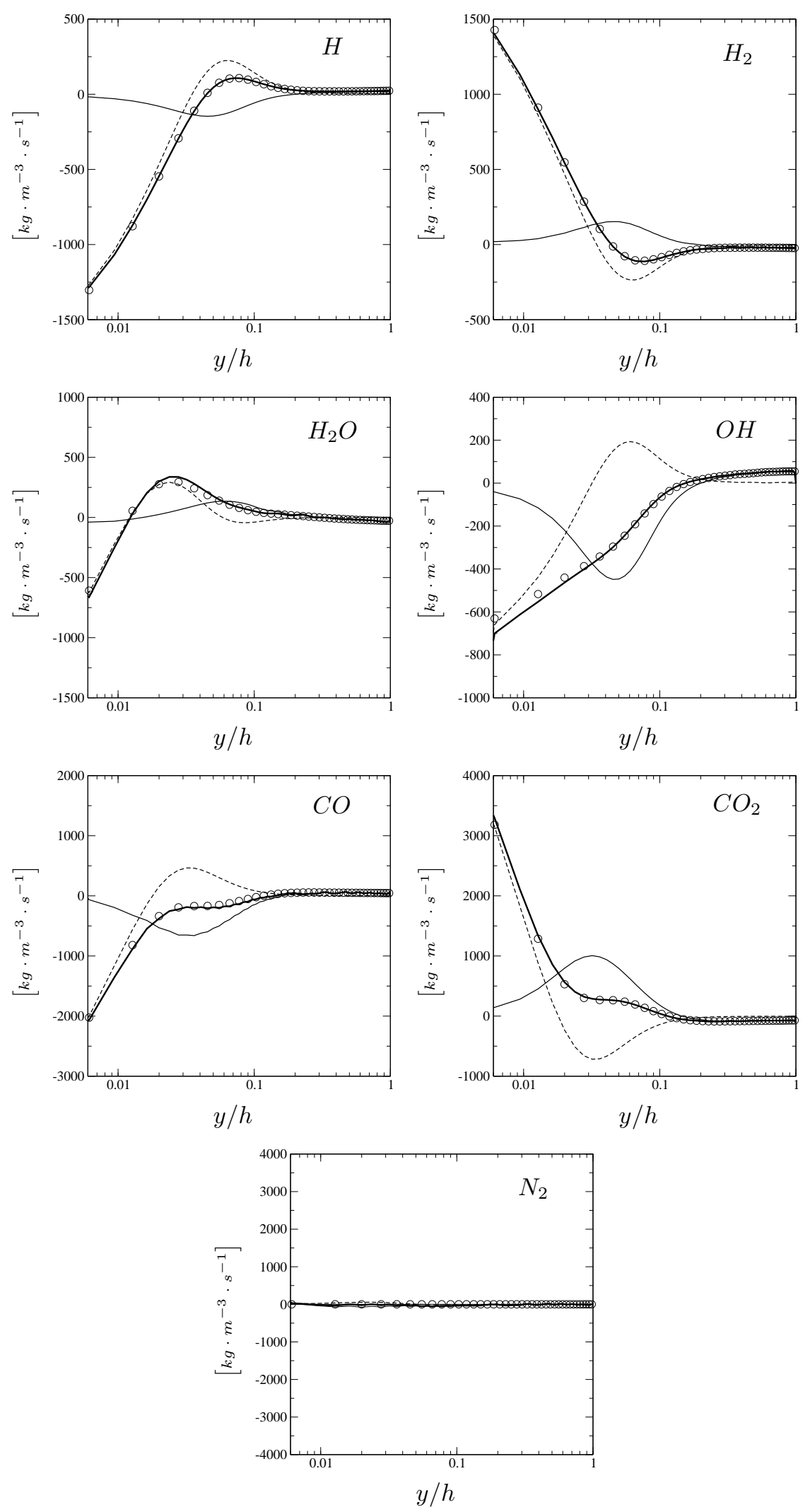

FIG. 6: Species mass fraction balances of case B (results for case D are not strictly identical but stay coherent with the analysis presented herein). $\quad-: d\left(\widetilde{\rho} \widetilde{v Y_{k}}\right) / d y ;----: d\left(\widetilde{\rho} \widetilde{V_{k, y} Y_{k}}\right) / d y$; $\longrightarrow: d\left(\bar{\rho}\left(\widetilde{v Y_{k}}+\widetilde{V_{k, y} Y_{k}}\right)\right) / d y ; \quad \circ: \overline{\omega_{k}}$. 

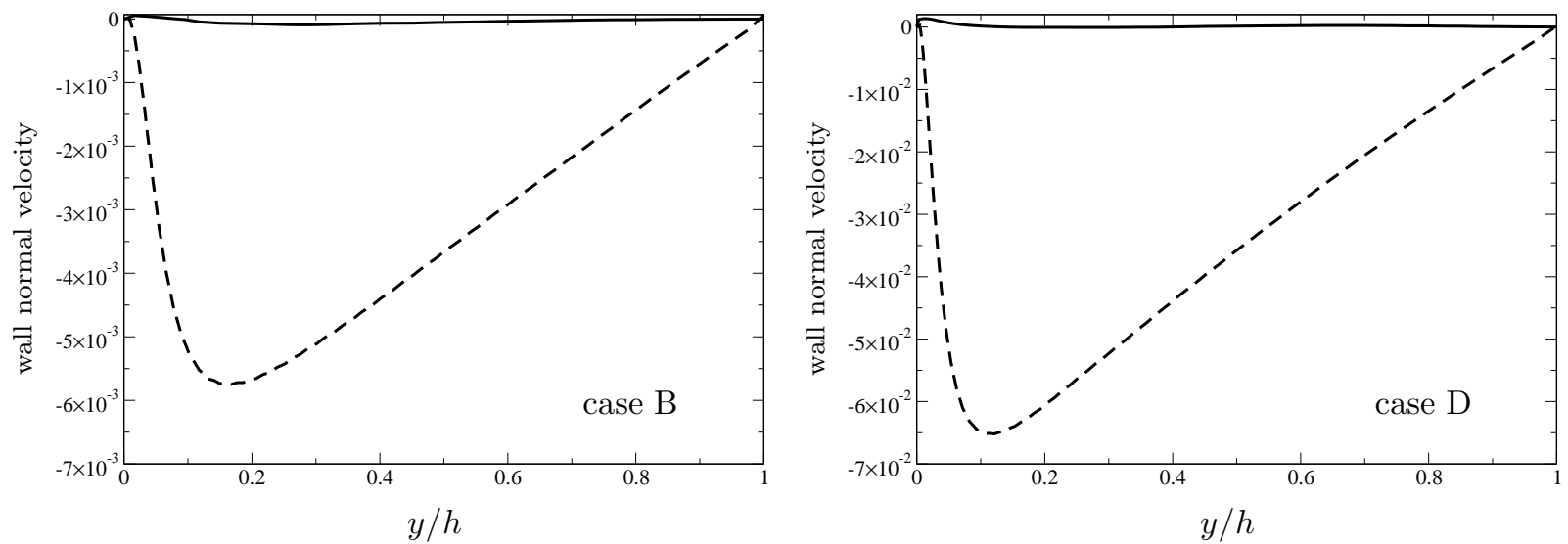

FIG. 7: Wall normal convective velocity profiles in wall units. Comparison between Reynolds and

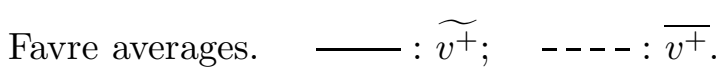

the wall, is well satisfied. This is shown in Fig. 7 that presents the Favre and Reynolds averaged wall normal convective velocity profiles scaled by the friction velocity: the maximum error observed on $\widetilde{v^{+}}$closure is around $0.01 \%$ for case $\mathrm{B}$ and $0.05 \%$ for case $\mathrm{D}$, whereas the maximum value of $\overline{v^{+}}$is around $0.6 \%$ for case $\mathrm{B}$ and $6.5 \%$ for case $\mathrm{D}$. Hence, because $\widetilde{v}=0$, any Favre averaged correlation of type $\widetilde{v f}$ between $v$ and any scalar $f$ can equivalently be written as $\widetilde{v^{\prime \prime} f^{\prime \prime}}$. This is not the case for Reynolds averaged correlations since $\bar{v} \neq 0$ because of the density variations.

Figure 6 suggests that the production term $\overline{\dot{\omega}_{k}}$ is mainly important in the region $y / h<0.2$ which indicates a strong activity of chemical reactions in the near wall region. It seems that the species production is null at the center of the channel in Fig. 6 but this is merely due to the graph scale effect. Indeed, Table VIII illustrates that the production term is not null at the centerline in both cases attesting that the effect of chemistry is present in the whole boundary layer.

Concerning the LHS terms, it is clear that neither one nor the other dominates the process of species conservation, whatever the species is. However, looking at the wall normal convective velocity (Fig. 7), the diffusion velocities (Fig. 11 and Fig. 12) and the species mass fractions (Fig. 9 and Fig. 10), two asymptotic regimes can be observed. In the fully turbulent region $(y / h>0.2)$, diffusion velocities vanish because gradients of species concentration asymptotically tend to zero. The behavior of the wall normal convective velocity is different: the gradient of $\bar{v}$ tend to a constant value. This explains why the species mass fraction 
TABLE VIII: Species production term at the centerline, $\overline{\dot{\omega}_{k, c}}$, scaled by the modulus of its wall value, $\left|\overline{\dot{\omega}_{k, w}}\right|$. Species $N_{2}$ is not shown because it is an inert species.

\begin{tabular}{|c|c|c|c|c|c|c|}
\hline \multirow[t]{2}{*}{ cases } & \multicolumn{6}{|c|}{$\overline{\dot{\omega}_{k, c}} /\left|\overline{\dot{\omega}_{k, w}}\right|$} \\
\hline & $\mathrm{H}_{2}$ & $H$ & $\mathrm{H}_{2} \mathrm{O}$ & $O H$ & $\mathrm{CO}_{2}$ & $C O$ \\
\hline B & $-1.52 \%$ & $1.6 \%$ & $-1.7 \%$ & $1.3 \%$ & $-1.1 \%$ & $1.1 \%$ \\
\hline $\mathrm{D}$ & $-12.7 \%$ & $14.1 \%$ & $-95.6 \%$ & $97.0 \%$ & $-19.7 \%$ & $19.7 \%$ \\
\hline
\end{tabular}

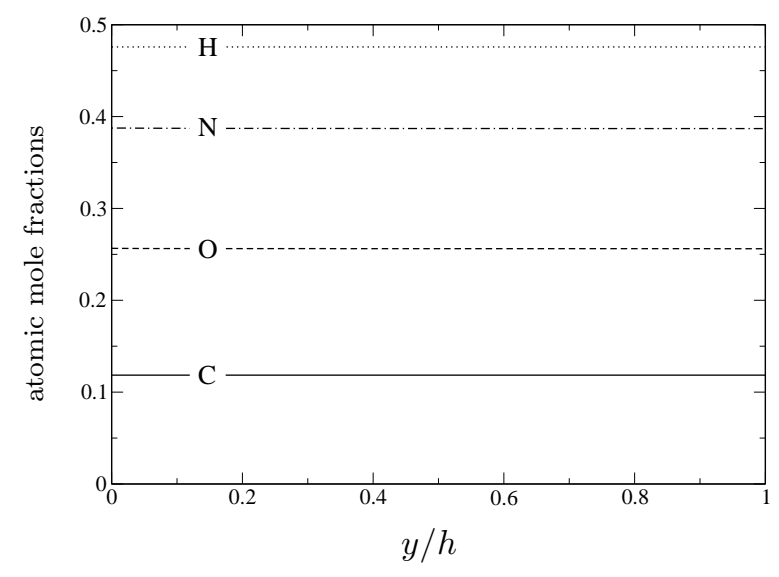

FIG. 8: Atomic composition of case B (profiles are identical for case D).

conservation is mainly led by the divergence of the wall normal convective flux in the fully turbulent region. In the vicinity of the wall $\left(y^{+}<10\right)$ the opposite is observed: the gradients of diffusion velocities are sharper than the one of the mean convective velocity and for this reason the mechanism of mass conservation tend to be led by species diffusion. We can summarize this analysis saying that the effect of species diffusion is important near the wall and lowered in the turbulent region. One thus expect that the contribution of species diffusion is negligible in the turbulent part, which will be confirmed in the forthcoming sections.

Figure 8 shows that the conservation of mass is virtually done at constant atomic composition. A chemical equilibrium state depends on three parameters: the number of atoms available, the thermodynamic pressure, and the temperature. Because no wall normal variation of atomic composition is observed for the four atoms present in the mixture $(C, H, O$ and $N$ ), the chemical equilibrium actually only depends on the temperature in the simulated 

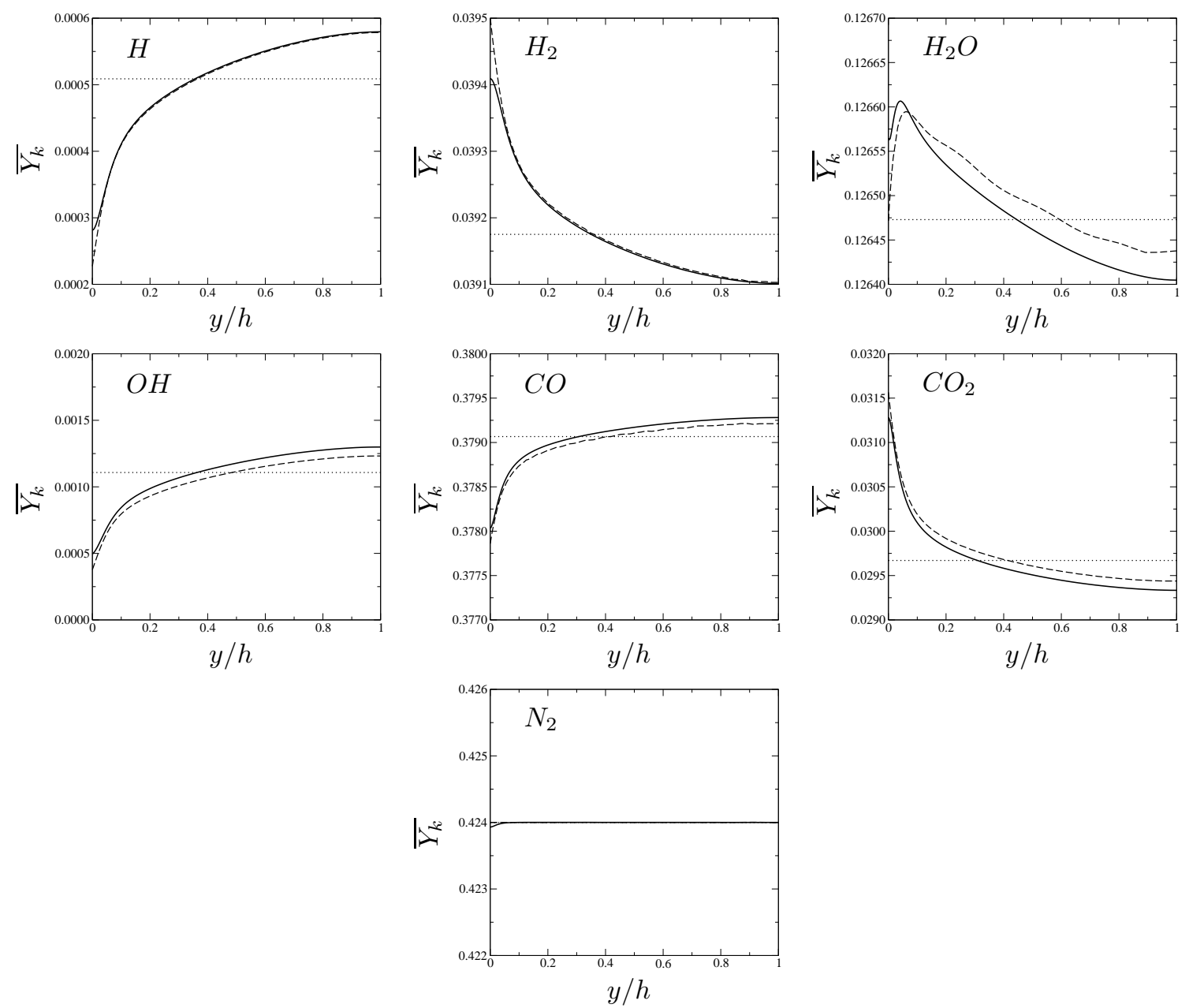

FIG. 9: Mass fraction profiles of cases A and B. : $Y_{k}$, case A (no chemistry); $: Y_{k}$ case B; ----: $Y_{k}^{\text {equil }}(T)$, equilibrium state computed with the mean temperature profile of case B (results obtained from CHEMKIN software).

turbulent boundary layer (one reminds that simulations of periodic channel flow are performed at constant thermodynamic pressure). This hypothesis has been successfully tested thanks to the EQUIL library of CHEMKIN software: equilibrium profiles that only depends on the temperature are generated by giving a unique mixture composition (here, the one at the centerline is chosen in order to be consistent with the wall model concept that uses variables taken in the outer part of the boundary layer) and the EQUIL solver is initialized by the mean temperature profiles of the simulations; the EQUIL library then rearranged the mixture composition solving a problem of the type "constant pressure and temperature". Results are shown in Fig. 9 and Fig. 10 that demonstrate the validity of this hypothesis: 

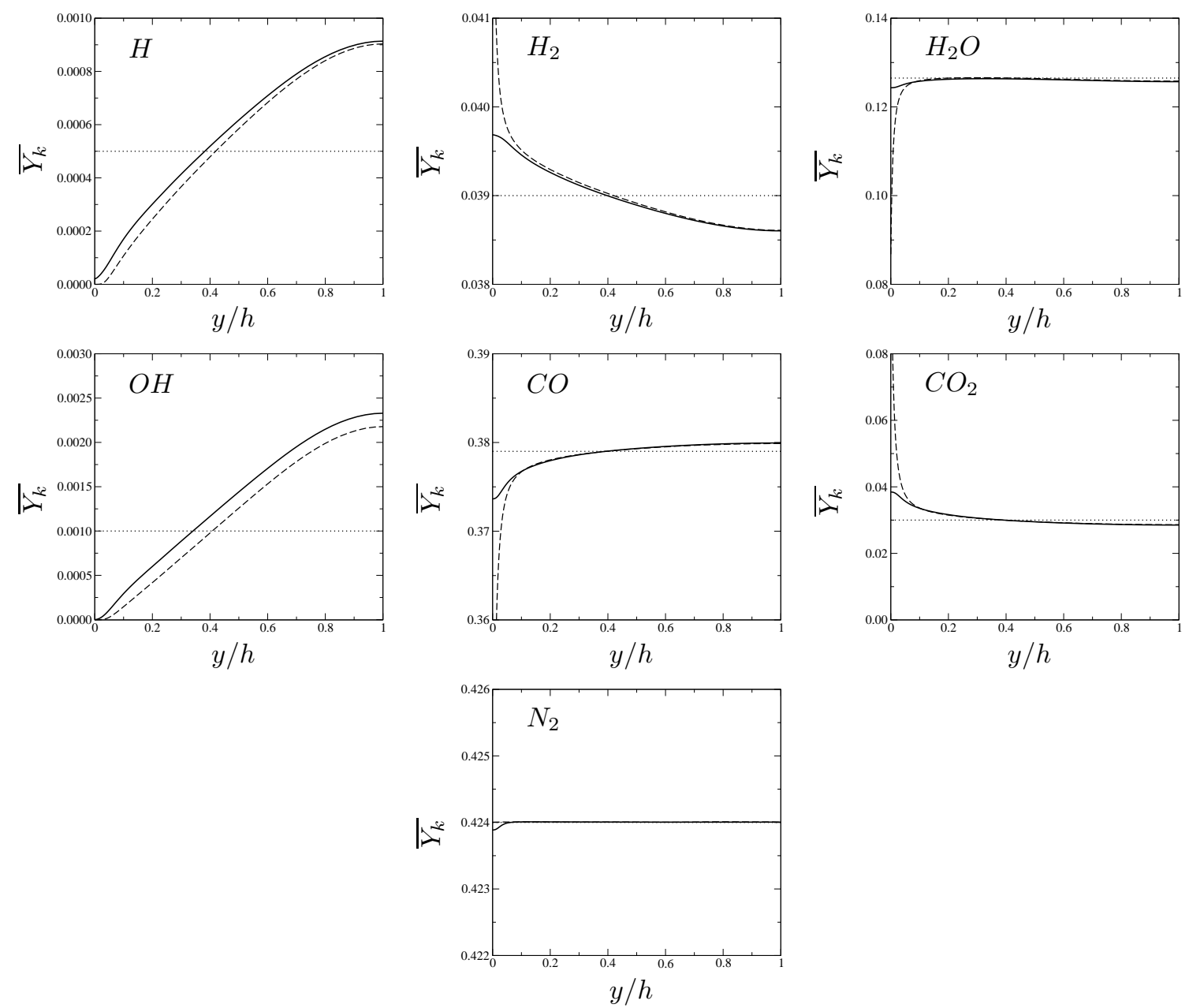

FIG. 10: Mass fraction profiles of the strong temperature gradient cases C and D. : $Y_{k}$ case $\mathrm{C}$ (no chemistry); — $: Y_{k}$, case D; ----: $Y_{k}^{\text {equil }}(T)$, equilibrium state computed with the mean temperature profile of case D (results obtained from CHEMKIN software).

$Y_{k} \approx Y_{k}^{\text {equil }}=Y_{k}^{\text {equil }}(T)$. One recalls that this condition is not imposed by the numerical resolution in the simulations since a chemical kinetic scheme composed of seven reactions is implemented in the code.

The profiles of the simulated cases and equilibrium state are not exactly similar at the wall which indicates that the characteristic time of diffusion tends to be shorter than the chemical characteristic time in this region. Moreover, the DNS/LES numerical solver imposes an impermeable boundary condition at the wall which is applied by imposing a null species flux in the wall normal direction, $\rho_{w} Y_{k, w} V_{k, y, w}=0$. With the Hirschfelder and Curtiss 

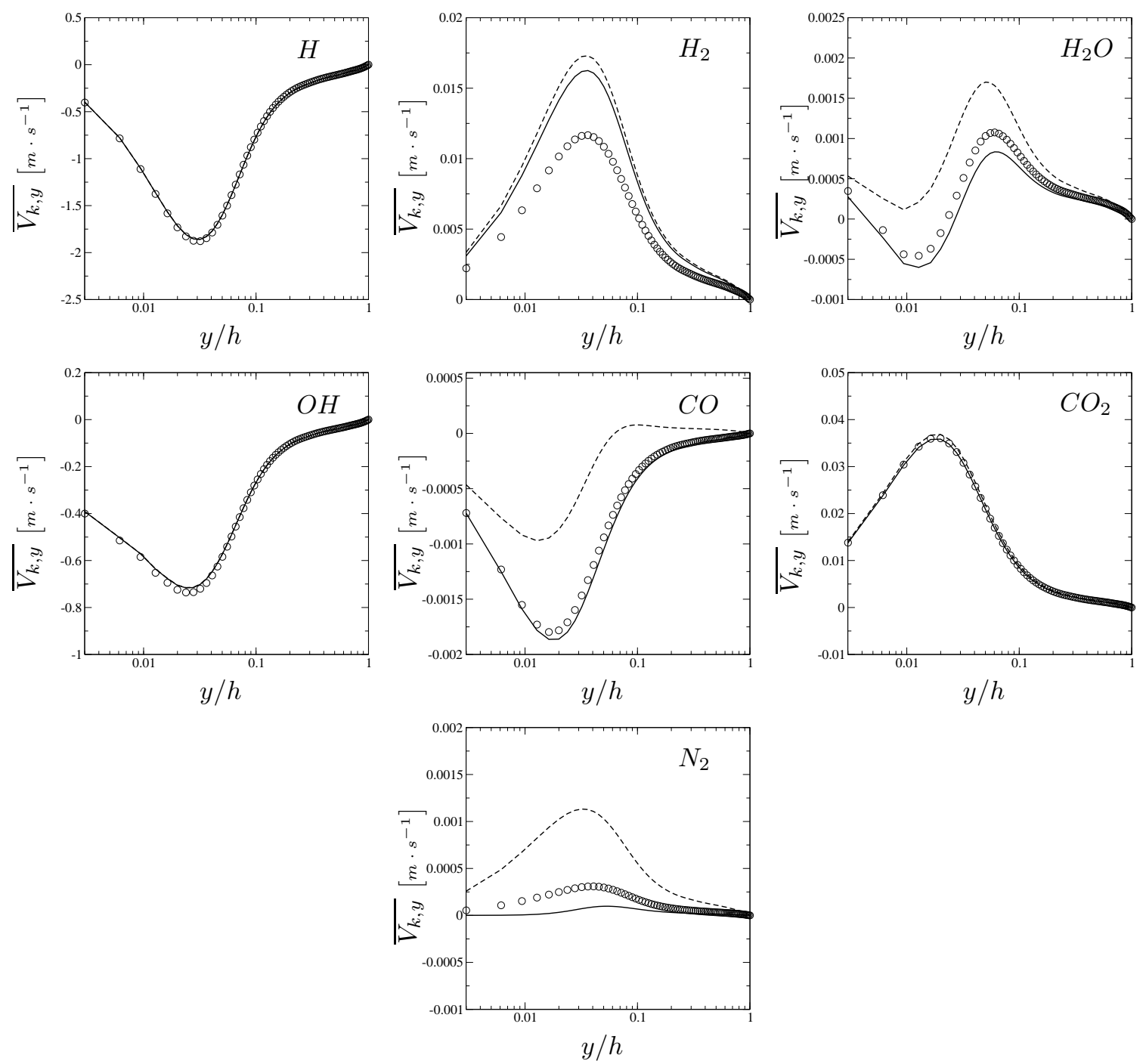

FIG. 11: Diffusion velocities of case B. $\quad----: \overline{V_{k, y}^{h c}} ; \quad-\quad-\overline{V_{k, y}}=\overline{V_{k, y}^{h c}}+\overline{V_{y}^{c o r}} ; \quad \circ: \overline{V_{k, y}^{\text {EGLIB }}}$. $\left(\overline{V_{k, y}^{h c}}\right.$ and $\overline{V_{k, y}}$ perfectly collapse for species $H$ and $O H$.)

approximation this implies:

$$
\left.\frac{\partial X_{k}}{\partial y}\right|_{w}=0
$$

a result visible on the DNS and LES profiles. Discrepancies on CHEMKIN profiles are observed in the vicinity of the wall $(y / h<0.05)$ because this impermeable boundary condition combined with the Hirschfelder and Curtiss approximation is not consistent with the chemical equilibrium assumption postulated above when the temperature gradient is not null. Indeed, making use of the equilibrium hypothesis, Eq. (19) can be rewritten as:

$$
\left.\left.\frac{d X_{k}}{d T}\right|_{e q} \frac{\partial T}{\partial y}\right|_{w}=0
$$



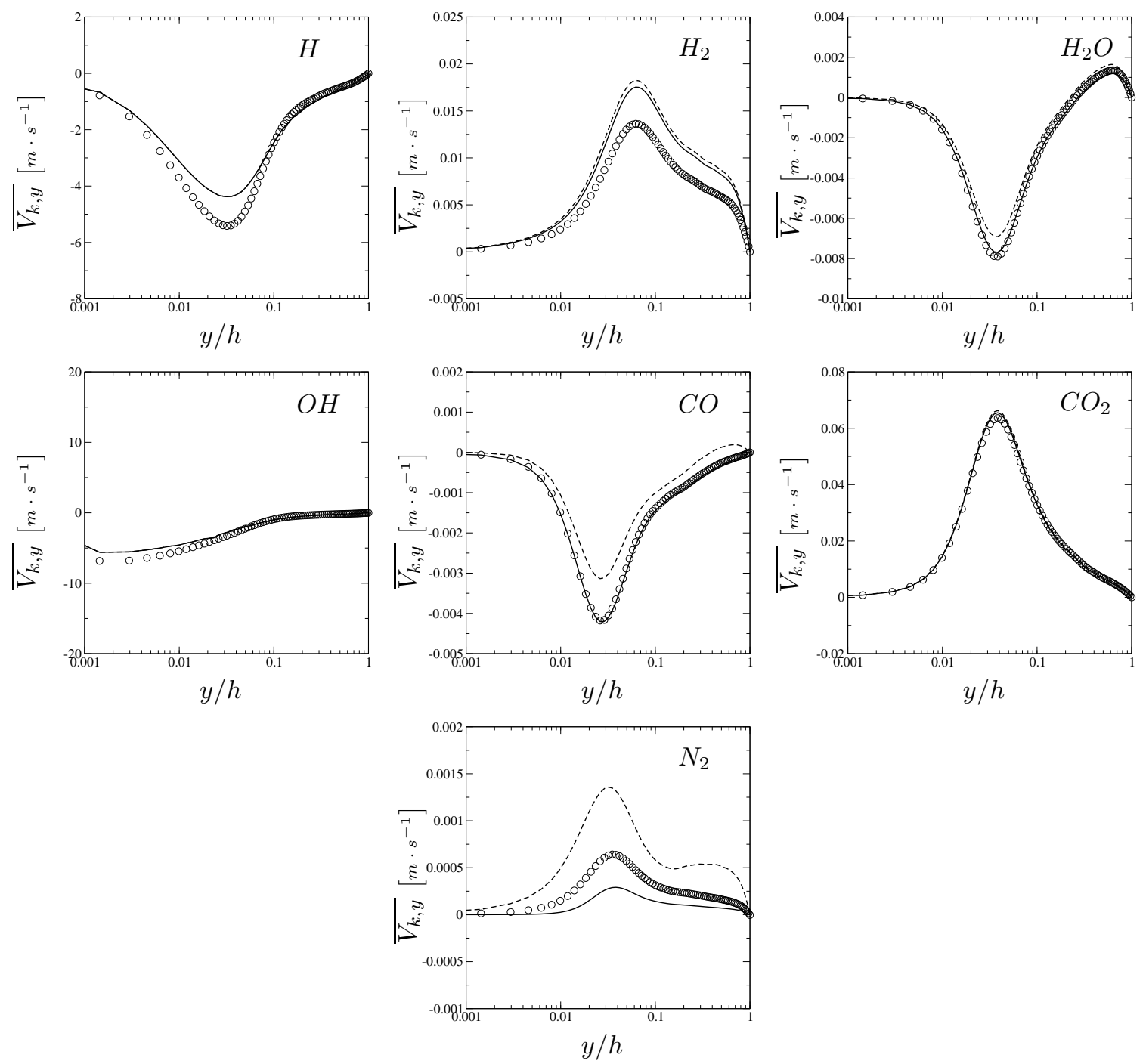

FIG. 12: Diffusion velocities of case D. $\quad----: \overline{V_{k, y}^{h c}} ; \quad-: \overline{V_{k, y}}=\overline{V_{k, y}^{h c}}+\overline{V_{y}^{c o r}} ; \quad \circ: \overline{V_{k, y}^{\text {EGLIB }}}$. $\left(\overline{V_{k, y}^{h c}}\right.$ and $\overline{V_{k, y}}$ perfectly collapse for species $H$ and $O H$.)

which is not verified by CHEMKIN because $d X_{k} /\left.d T\right|_{e q} \neq 0$ and $\partial T /\left.\partial y\right|_{w} \neq 0$. Note that if the Soret effect were accounted for to evaluate diffusion velocities, we would not have observed such an inconsistency at the wall. In this case another term arises in Eq. (19) and Eq. (20) so that $\rho_{w} Y_{k, w} V_{k, y, w}=0$ does not imply $\partial X_{k} /\left.\partial y\right|_{w}=0$. However, differences between the two profiles remains small for each species and the chemical equilibrium hypothesis is well justified for $95 \%$ of the profile, notably in the turbulent region.

To evaluate the global behavior of the Hirschfelder and Curtiss model with correction velocity, $\overline{V_{y}^{h c}}$ of the simulations has been confronted in Fig. 11 and Fig. 12 to the effective 
diffusion velocities of the simulations, $\overline{V_{k, y}}=\overline{V_{k, y}^{h c}}+\overline{V_{y}^{c o r}}$, and to the diffusion velocities obtained by solving the full system Eq. (8) thanks to a post-processing with the EGLIB library $[68,69]$ which solves the transport linear system with direct inversion. The latter are noted $\overline{V_{k, y}^{\text {EGLIB }}}$ and represent the reference data. Except for species $H_{2}$ and $N_{2}$, we observe a very good agreement between the effective diffusion velocities of the simulations and the ones computed a priori with EGLIB. Moreover, comparing $\overline{V_{k, y}}$ and $\overline{V_{k, y}^{h c}}$ we see that the correction velocity modifies the Hirschfelder and Curtiss diffusion velocities, $\overline{V_{k, y}^{h c}}$, in the good direction. This is clearly visible looking at profiles of species $\mathrm{H}_{2} \mathrm{O}, \mathrm{CO}$ and $\mathrm{CO}_{2}$. Hence, the correction velocity is not intrusive for the computation and the model retained in the numerical solver to simulate mass diffusion is acceptable.

\section{B. Momentum balance}

Since the momentum conservation equation, Eq. (3), is not directly influenced by the mixture composition, the only possible changes induced by chemical reactions on the momentum balance arise indirectly from the coupling with the mass and energy equations. The average procedure applied to the equation for momentum conservation in the streamwise direction leads to the following reduced form:

$$
\frac{d}{d y}\left(\overline{\mu \frac{d u}{d y}}-\bar{\rho} \widetilde{u^{\prime \prime} v^{\prime \prime}}\right)=-\mathcal{S}_{x}
$$

Figure 13 presents the integrated form of the latter equation which is equivalent to a balance of shear stress. The total shear stress is linear through the channel flow, the -1 slope being related to the imposed source term in the streamwise momentum equation. This illustrates that the feature predicted by Eq. (21) is well reproduced by numerical results, attesting that the data sample is large enough to compute realistic statistics. Since the streamwise momentum source term simulates a constant pressure gradient in the boundary layer, this slope would not exist if the flow were a zero pressure gradient one. Hence, the total shear stress would be constant all along the boundary layer. This comment will be fully discussed in the modeling section of this paper, Sec. IV.

Note also that in Fig. 13 the laminar shear stress refers to the term $\overline{\mu d u / d y}$ which, strictly speaking, contains some turbulent contributions, $\overline{\mu^{\prime} d u^{\prime} / d y}$. However, it is shown in the same figure that the latter turbulent term is negligible because the laminar shear stress 

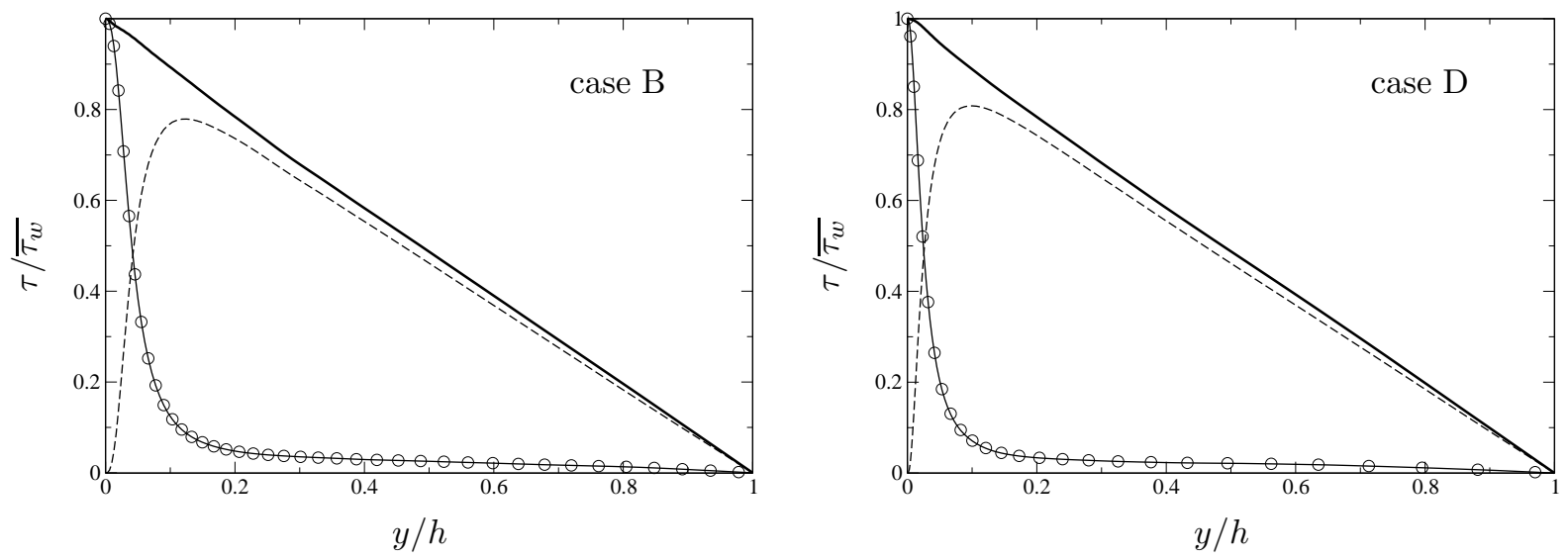

FIG. 13: Momentum conservation balance scaled by $\tau_{w}$.

- : laminar shear stress, $\overline{\mu \frac{d u}{d y}}$; ---- : turbulent shear stress, $-\bar{\rho} \widetilde{u^{\prime \prime} v^{\prime \prime}}$; : Total shear stress, $\overline{\mu \frac{d u}{d y}}-\bar{\rho} \widetilde{u^{\prime \prime} v^{\prime \prime}} ; \quad \circ: \bar{\mu} \frac{\overline{d u}}{d y}$.
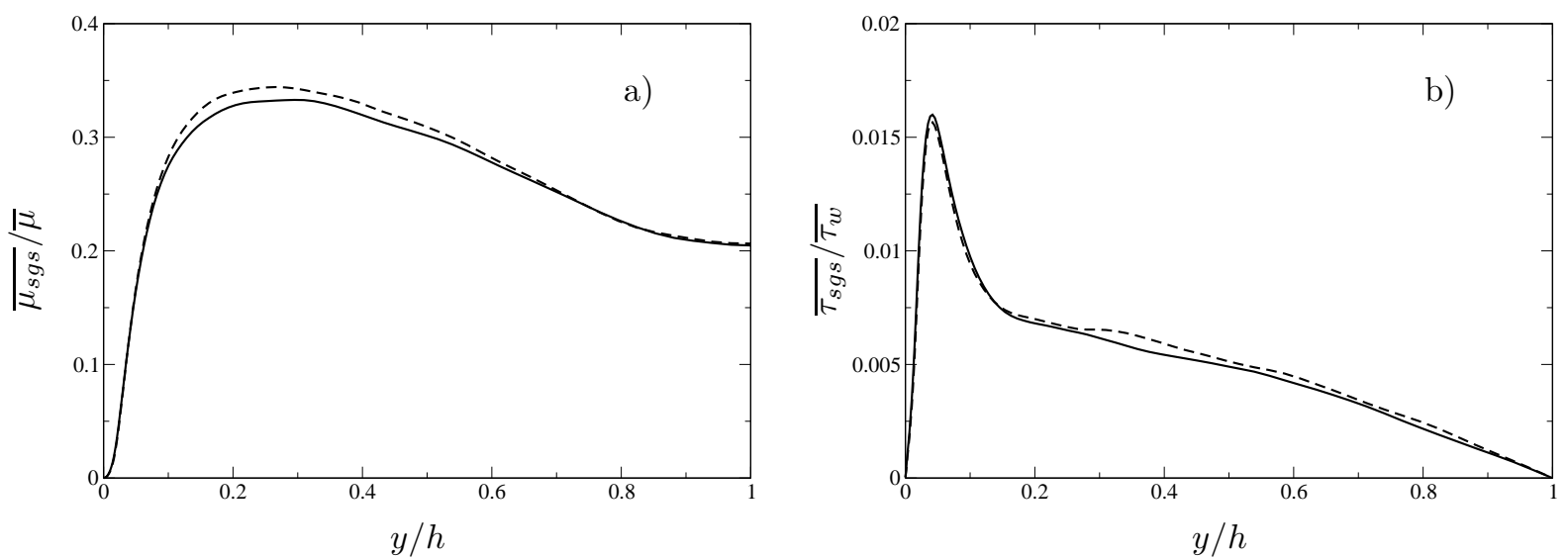

FIG. 14: Subgrid-scale contributions for LES cases C and D: a) subgrid dynamic viscosity, $\overline{\mu_{s g s}}$, scaled by its laminar value; b) subgrid shear stress, $\overline{\tau_{s g s}}$, scaled by the total wall shear stress. - - - - : case C; — : case D.

is perfectly approximated by the relation $\overline{\mu d u / d y}$. This classical result for boundary layer flows[13] is thus confirmed for the compressible reacting general case.

As illustrated in Fig. 14, the contribution of the subgrid-scale model for LES cases C and $\mathrm{D}$ is negligible: $\overline{\mu_{s g s}} / \bar{\mu} \approx 0.3$ and $\overline{\tau_{s g s}} / \overline{\tau_{w}}$ never exceeds $1.6 \%$. The subgrid shear stress is thus not shown in the momentum conservation balance Fig. 13. For the same reason, the subgrid terms will not be shown in the heat flux balances (Fig. 22 and Fig. 23). 

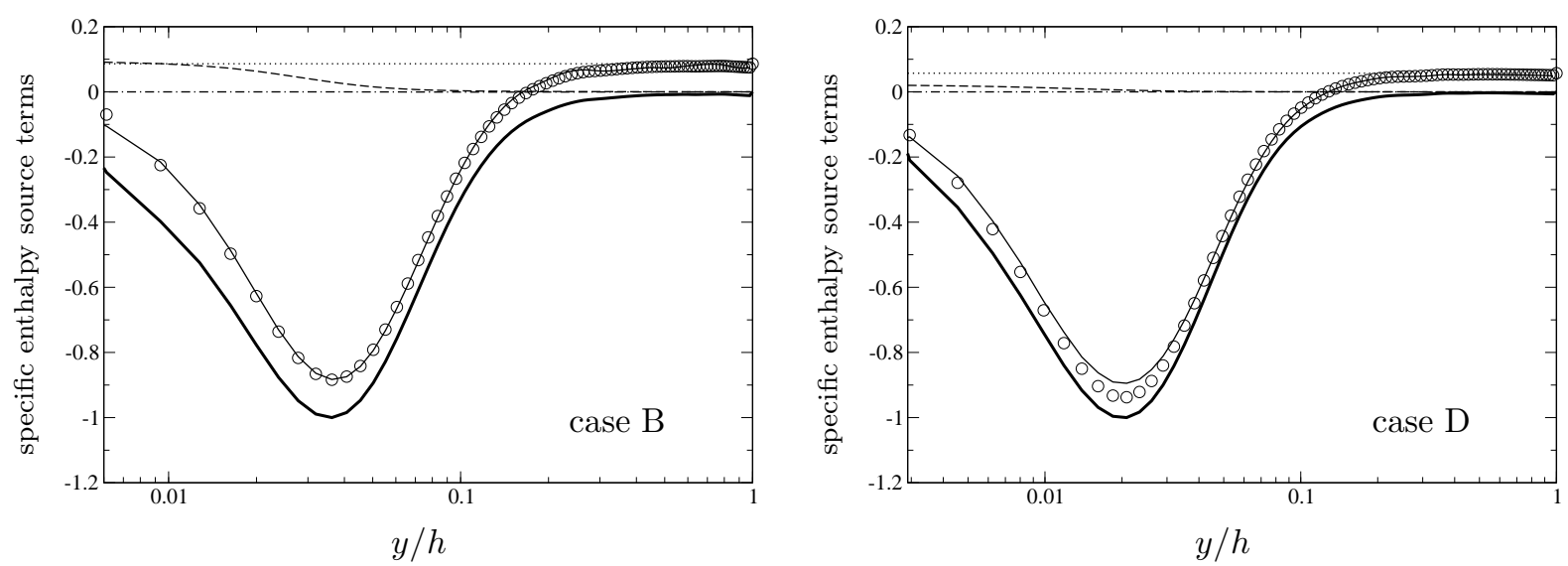

FIG. 15: Specific enthalpy balance scaled by $\left|\overline{d q_{y} / d y}\right|_{\text {max }}$, for cases B and D. — : molecular diffusion, $-\frac{\overline{d q_{y}}}{d y} ; \quad$........ : energy source term, $\overline{\mathcal{Q}} ; \quad$---- : viscous term, $\overline{\tau_{i y} \frac{d u_{i}}{d y}} ; \quad$ - - - : power of pressure forces, $\overline{v \frac{d p}{d y}} ; \quad$ ○: total of RHS terms of Eq. (23); ㄴ : turbulent diffusion, $\frac{d}{d y}\left(\bar{\rho} \widetilde{v^{\prime \prime} h^{\prime \prime}}\right)$.

\section{Energy balance}

In this section the specific enthalpy conservation equation (22) has been retained for practical reasons:

$$
\rho \frac{D h}{D t}=\frac{D p}{D t}+\tau_{i j} \frac{\partial u_{i}}{\partial x_{j}}-\frac{\partial q_{i}}{\partial x_{i}}+\mathcal{Q}
$$

with summation on $i$ and $j$-subscripted variables. After the statistical procedure has been applied, one obtains the following equation:

$$
\frac{d}{d y}\left(\bar{\rho} \widetilde{v^{\prime \prime} h^{\prime \prime}}\right)=\overline{v \frac{d p}{d y}}+\overline{\tau_{i y} \frac{d u_{i}}{d y}}-\overline{\frac{d q_{y}}{d y}}+\overline{\mathcal{Q}}
$$

Note that all the terms presented in Fig. 15 are voluntarily not fully decomposed for clarity and because the main goal here is to show that the balance is well closed. For instance, the specific enthalpy and the wall normal molecular heat flux could have been decomposed into $h=\int_{T_{0}}^{T} C_{p} d T+\sum_{k} \Delta h_{f, k}^{0} Y_{k}$ and $q_{y}=-\lambda \frac{d T}{d y}+\rho \sum_{k} h_{k} Y_{k} V_{k, y}$, respectively. Hence, the effects of chemistry on the energy conservation mechanisms are not explicitly visible in this balance, although they are present.

Figure 15 shows that the power of the pressure forces, $\overline{v d p / d y}$, is negligible in front of any other term. Moreover, the viscous effect term $\overline{\tau_{i y} d u_{i} / d y}$ (which is not the viscous heating, generally referred to as $d \overline{\tau_{i y} u_{i}} / d y$ ) is clearly negligible in the region $y / h>0.05$ because the flow is at low Mach number. In the vicinity of the wall, the viscous effect is not strictly 
null but its contribution sharply vanishes in front of the molecular diffusion term. As a consequence, it is not necessary to include this term in a low Mach number wall model. Note also in Fig. 15 that the energy source term, $\overline{\mathcal{Q}}$, is well constant as described in Sec. II.

\section{Comment on the Dufour effect}

One recalls that the computations have been performed neglecting the Dufour part of the molecular heat flux, $q_{i}$. Indeed, if the Dufour effect were considered, Eq. (7) would have been replaced by the following one:

$$
q_{i}=\underbrace{-\lambda \frac{\partial T}{\partial x_{i}}}_{\text {Fourier term }}+\underbrace{\rho \sum_{k} h_{k} Y_{k} V_{k, i}}_{\text {species diffusion }}+\underbrace{p \sum_{k} \chi_{k} V_{k, i}}_{\text {Dufour term }}
$$

where $\chi_{k}$ are the species thermal diffusion ratios. To quantify the importance of the Dufour term in the simulations, the EGLIB library $[68,69]$ has been used to evaluate the thermal diffusion ratios and the Dufour part has been rebuilt a priori using the data of the DNS/LES for $p$ and $V_{k, i}$. The resulting profile of the wall normal component of the Dufour term, $\overline{q_{y, \text { Dufour }}}$, has been plotted in Fig. 16 for cases B and D. This figure illustrates that the Dufour part of the molecular heat flux is negligible $\left(\overline{q_{y, \text { Dufour }}}\right.$ does not exceeds $0.25 \%$ of the total heat flux at the wall for case B and $0.025 \%$ for case D) which confirms the assumption made to perform the simulations. Because the thermal diffusion ratios depends on the mixture composition, this conclusion stands for the present study but would be verified for any other mixture. However, the Dufour effect might be negligible for $95 \%$ of the applications.

The mass/momentum/energy balances have been investigated in this section, leading to first approximations concerning the conservation of energy (the power of pressure forces, the viscous effect terms and the Dufour effect are negligible). Following the idea of integrating the reacting Navier-Stokes equations in order to derive relevant expressions for the assessment of wall fluxes, it is shown in the forthcoming section how the remaining terms can be modeled. 


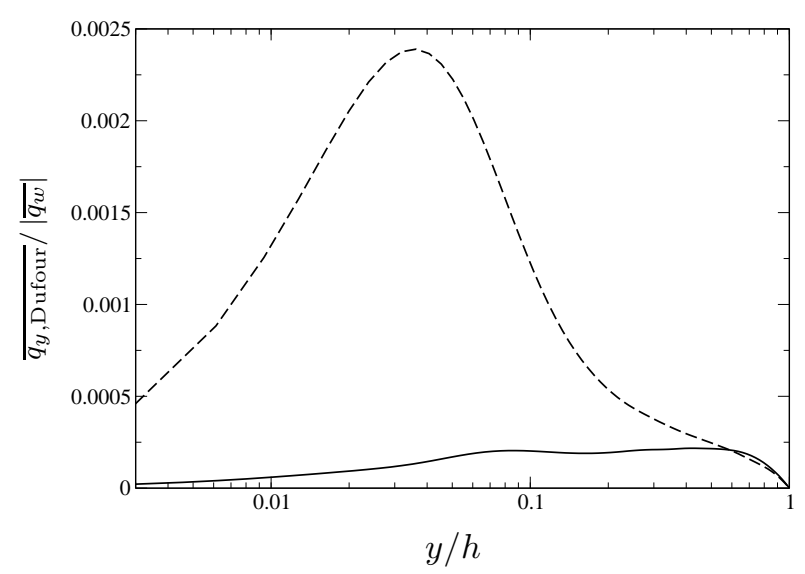

FIG. 16: Wall normal value of the Dufour heat flux computed a priori thanks to EGLIB library. Results are scaled by the total heat flux at the wall. - ----: case B; —

\section{DEVELOPMENT OF WALL MODELS}

Starting from the general flow equations, one seeks for two independent equations of the type:

$$
\frac{d f}{d y}=F ; \quad \frac{d g}{d y}=G
$$

where $f$ and $g$ stand for the total flux of momentum and energy in the $y$-direction respectively. In their general formulation, the two functions $f$ and $g$ are dependent on the following set of variables: $\left(y, \tau_{w}, q_{w}, u_{\text {out }}, T_{\text {out }}, T_{w}, Y_{k, \text { out }}, Y_{k, w}\right)$, where $w$-subscripted variables refer to wall quantities and out-subscripted ones refer to outer flow conditions. If $F$ and $G$ are simple functions of $y$, Eq. (25) can be integrated over space to generate a $2 \times 2$ non-linear system of equations with $q_{w}$ and $\tau_{w}$ the unknowns. In other words, integrating Eq. (25) leads to a law-of-the-wall which can be used to assess the momentum and energy fluxes at the wall from the wall/outer flow conditions $\left(u_{\text {out }}, T_{\text {out }}, T_{w}, Y_{k, \text { out }}\right.$ and $\left.Y_{k, w}\right)$. The simplest case would be $F=G=0$ but the case $F=F_{0}$ and $G=G_{0}$ where $F_{0}$ and $G_{0}$ are constant values also leads to a suitable wall model. On the contrary, if $F$ and/or $G$ are/is unknown function of y, Eq. (25) cannot be used to obtain a law-of-the-wall, as illustrated in Fig. 17.

The framework of this section follows five main assumptions for the flow: 1) the Reynolds number is infinite so that no wake function will be considered to describe the outer part of the boundary layer; 2) the Mach number is null; 3) no streamwise pressure gradients; 4) no blowing or suction at the wall surface; 5) no roughness effect. 


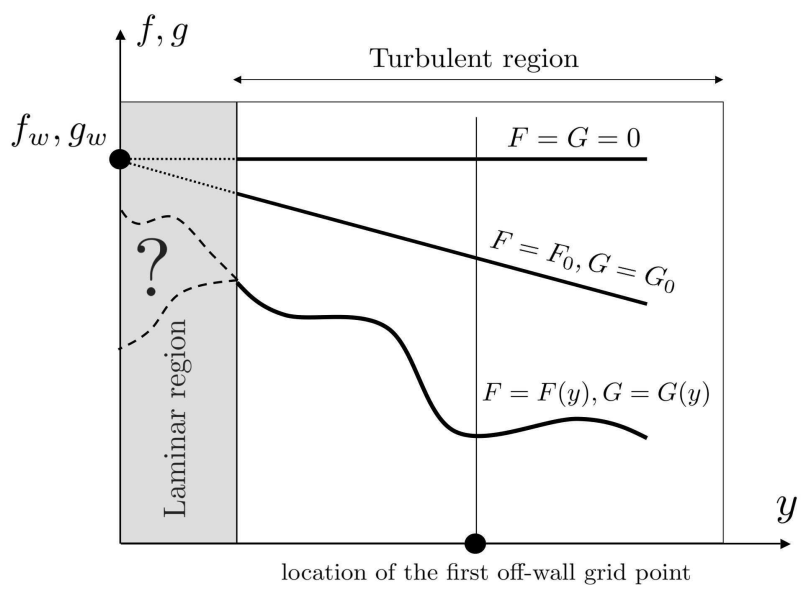

FIG. 17: Illustration of wall flux estimation knowing data from the first off-wall grid point located in the turbulent region ( $f$ represents the total shear stress, $g$ the total heat flux).

\section{Finding a function for the total shear stress}

When we have analyzed the momentum balance (Sec. IIIB), we have seen that the laminar shear stress $\overline{\mu d u / d y}$ is almost perfectly approximated by $\bar{\mu} d \bar{u} / d y$. The equation for the total shear stress thus takes the form:

$$
\frac{d \overline{\tau_{t o t}}}{d y} \approx \frac{d}{d y}\left(\bar{\mu} \frac{d \bar{u}}{d y}-\bar{\rho} \widetilde{u^{\prime \prime} v^{\prime \prime}}\right)=-\mathcal{S}_{x}
$$

Note that with respect to the form of equation we are looking for, Eq. (25), $F$ is not null as desired but is equal to a constant value, $-\mathcal{S}_{x}$. We have seen that this term was a pressure gradient like contribution to the momentum conservation which gives the slope for the total shear stress in Fig. 13. Since we seek for a wall model valid in the zero/small pressure gradient regime, this term is set to zero in what follows. The non-dimensionalized streamwise pressure gradient, $p_{x}^{+}=\left(\nu_{w} / \rho_{w} u_{\tau}^{3}\right)(d p / d x)$, is equal to $p_{x}^{+}=-1 / R e_{\tau}$ for a periodic channel flow which implies that $p_{x}^{+} \approx-0.003$ for cases $\mathrm{A}$ and $\mathrm{B}$, and $p_{x}^{+} \approx-0.001$ for cases $\mathrm{C}$ and D. As explained by Nickels[48], this value is not strong enough to influence the behavior of the laminar and turbulent shear stresses (the pressure gradient should have an influence if $\left.\left|p_{x}^{+}\right|>0.005\right)$. This justifies the development of wall model for zero streamwise pressure gradient from our database.

We thus recover the classical formulation:

$$
\frac{d \overline{\tau_{t o t}}}{d y} \approx \frac{d}{d y}\left(\bar{\mu} \frac{d \bar{u}}{d y}-\bar{\rho} \widetilde{u^{\prime \prime} v^{\prime \prime}}\right)=0
$$




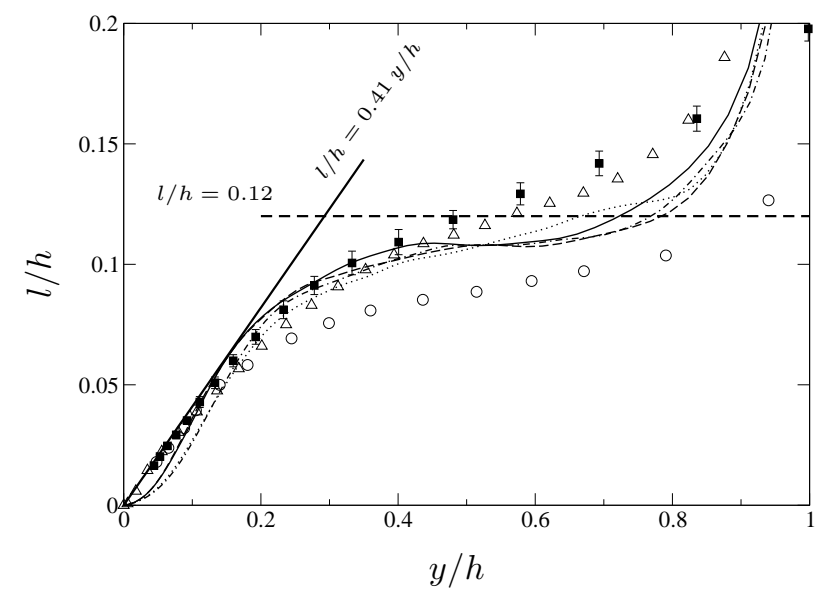

FIG. 18: Mixing-length, computed as $l=\left(-\widetilde{u^{\prime \prime} v^{\prime \prime}}\right)^{1 / 2} /(d \bar{u} / d y)$, scaled by the channel half-height. - - - : case A; …... : case B; ---- : case C; — : case D; $\triangle$ : Hoyas and Jiménez[35], $R e_{\tau}=2000$; : Österlund et al.[53], error bars representing a 95\% confidence interval; O: Andersen et al. [76].

where the turbulent shear stress $-\bar{\rho} \widetilde{u^{\prime \prime} v^{\prime \prime}}$ can be approximated with the classical Boussinesq assumption combined with a Prandtl mixing-length model for the turbulent dynamic viscosity, $\mu_{t}$. Hence, the turbulent shear stress can be modeled by:

$$
-\bar{\rho} \widetilde{u^{\prime \prime} v^{\prime \prime}} \approx \mu_{t} \frac{d \bar{u}}{d y} \approx \bar{\rho} l^{2}\left(\frac{d \bar{u}}{d y}\right)^{2}
$$

where $l$ denotes the mixing-length. For near wall flows, it has been shown that the mixinglength scales with the distance to the wall following the relation $l=\kappa y$, with $\kappa$ the von Kármán constant whose value is usually assumed to be 0.41 . This value has been criticized because some studies have shown that $\kappa$ can change with the Reynolds number. This is not consistent with the classical log-law, $u^{+}=1 / \kappa \ln y^{+}+C$, which is not a function of Re. However, the experiments of Österlund et al.[53] have raised this confusion showing that the classical log-law formulation is no longer $R e$-dependent if $\kappa$ is set to 0.38 and the additive constant, $C$, to 4.1 (this result has latter been confirmed by the study of Buschmann and Gad-el-Hak[54]). Nevertheless, the values retained in this study for $\kappa$ and $C$ will remain the most classical ones, $\kappa=0.41$ and $C=5.5$ for wall-bounded flows $(C=5.2$ for external boundary layer), mainly because this has little influence on the final prediction of $u^{+}$. Indeed, a computation of the error $\left(u_{O}^{+}-u_{c}^{+}\right) / u_{c}^{+}, u_{O}^{+}$being the value advocated by Österlund et al. and $u_{c}^{+}$the classical one, leads to an expression that depends on $y^{+}$: 
$\left(u_{O}^{+}-u_{c}^{+}\right) / u_{c}^{+}=\left(0.19 \ln y^{+}-1.4\right) /\left(2.44 \ln y^{+}+5.5\right)$; the error does not exceed $5 \%$ for $50<y^{+}<10^{8}$ (the two profiles are shown in Fig. 20 untill $y^{+}=10^{3}$ ).

Figure 18 demonstrates the efficiency of the relation $l=\kappa y$. Results from cases A, B, $\mathrm{C}$ and $\mathrm{D}$ are confronted to the numerical result of Hoyas and Jiménez[35] as well as to the experimental measurements of Andersen et al.[76] and Österlund et al.[53]. As in the classical theory[77], we first note that the mixing length scales reasonably well with the channel half-height (or equivalently the boundary layer height for external flows) since all the profiles collapse. Moreover, our results are consistent with the ones of the high friction Reynolds number channel flow of Hoyas and Jiménez[35] and with the external boundary layer of Österlund et al.[53]. The profile of Andersen et al.[76] for zero pressure gradient shows some discrepancies with others profiles but the trend remains unchanged: the von Kármán constant reproduces the mixing-length fairly well up to $y / h \approx 0.2$, with or without chemical reactions and significant heat transfer. For $y / h>0.2$, the mixing-length is no longer dependent on the distance from the wall and tends to a constant value $(l / h \approx 0.12$ from our simulations) which is also a classical result[77]. The sudden increase of $l$ in the center region is due to $d \bar{u} / d y$ that tends to zero at the centerline.

Figure 19 shows the mixing length as a function of $y^{+}$and illustrates that the collapse with the von Kármán model is enhanced when the Reynolds number increases. This supports the use of the relation $l=\kappa y$ for developing wall models which are designed to handle large Reynolds number flows. Moreover this figure demonstrates that when the friction Reynolds number is too weak, $R e_{\tau}=180$ for instance, the simulation is not representative of an infinite Reynolds number boundary layer because the mixing length never matches with the von Kármán model. Hence, the higher the $R e_{\tau}$ of the simulation is, the better is the validation of the wall model with a priori tests.

Thus, the use of the von Kármán constant is justified to develop wall models. Integrating Eq. (27) over space, one obtains:

$$
\overline{\tau_{w}} \approx \bar{\mu} \frac{d \bar{u}}{d y}+\bar{\rho}(\kappa y)^{2}\left(\frac{d \bar{u}}{d y}\right)^{2}
$$

where the constant total shear stress $\overline{\tau_{t o t}}$ has been replaced by its wall value $\overline{\tau_{w}}$ because Eq. (27) indicates that the total shear stress is constant throughout the wall region, i.e. $\overline{\tau_{t o t}} \equiv \overline{\tau_{w}}$. Neglecting the laminar contribution, because in the context of wall modeling the first off-wall point has to be in the fully turbulent region, we finally recover the classical 


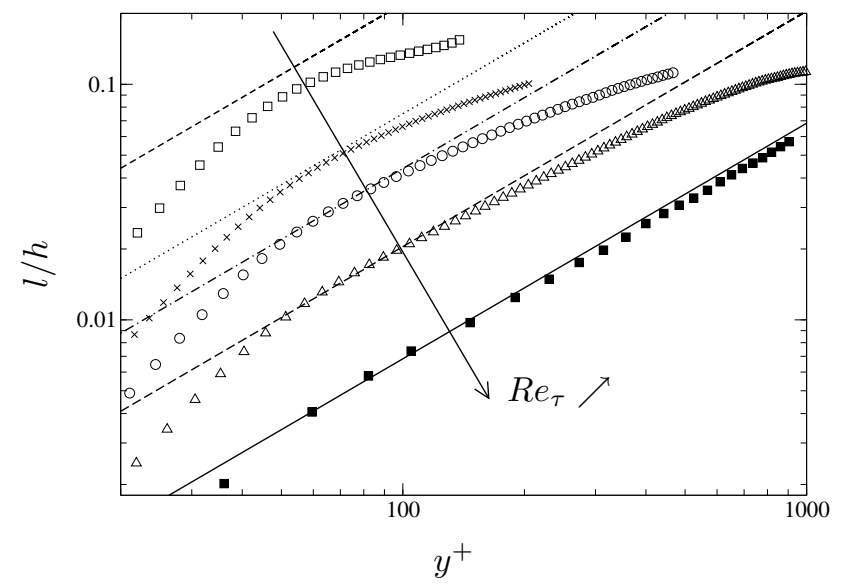

FIG. 19: Mixing-length scaled by the channel half-height as a function of $y^{+}$. Symbols represent results from the DNS database of del Álamo et al.[78, 79] and Hoyas et al.[35] (URL: http://torroja.dmt.upm.es/ftp/channels/), and from measurements of Österlund et al.[53]. Straight lines refer to the classical scaling $l=\kappa y$ with $\kappa=0.41 . \quad(\square,----)$ : Ref. [78], $R e_{\tau}=180$; $(\times, \cdots \cdots .$.$) : Ref. [78], R e_{\tau}=550 ; \quad(O,---)$ : Ref. [79], $R e_{\tau}=950 ; \quad(\triangle,---)$ : Ref. [35], $R e_{\tau}=2000 ; \quad(\boldsymbol{\square},-)$ : experiments of Österlund et al.[53];

form:

$$
\overline{\tau_{w}} \approx \bar{\rho}(\kappa y)^{2}\left(\frac{d \bar{u}}{d y}\right)^{2}
$$

or equivalently in wall units:

$$
\left(\frac{\bar{\rho}}{\overline{\rho_{w}}}\right)^{1 / 2} d u^{+} \approx \frac{d y^{+}}{\kappa y^{+}}
$$

After integration, the latter equation leads to the well-known Van Driest transformation[22]:

$$
u_{V D}^{+}=\int_{0}^{u^{+}}\left(\frac{\bar{\rho}}{\overline{\rho_{w}}}\right)^{1 / 2} d u^{+} \approx \frac{1}{\kappa} \ln y^{+}+C
$$

The efficiency of this scaling has been mainly demonstrated for high Mach number flows[23, 25-27] (up to Mach $\approx 8$ ) and the case of significant heat transfer but low Mach number has received little attention by experimenters. However, since the transformation is based on the assumption that turbulence structure is unaltered by large temperature variations it should be as valid at low Mach numbers as at high ones. Indeed, Fig. 20 shows that the results of our simulations collapse quite well in the plane $\left(y^{+}, u_{V D}^{+}\right)$, supporting the usefulness of the Van Driest transformation to retrieve the classical logarithmic law of the wall in the case of strong temperature gradient $\left(T_{c} / T_{w} \approx 3\right)$ and low Mach number, with or 

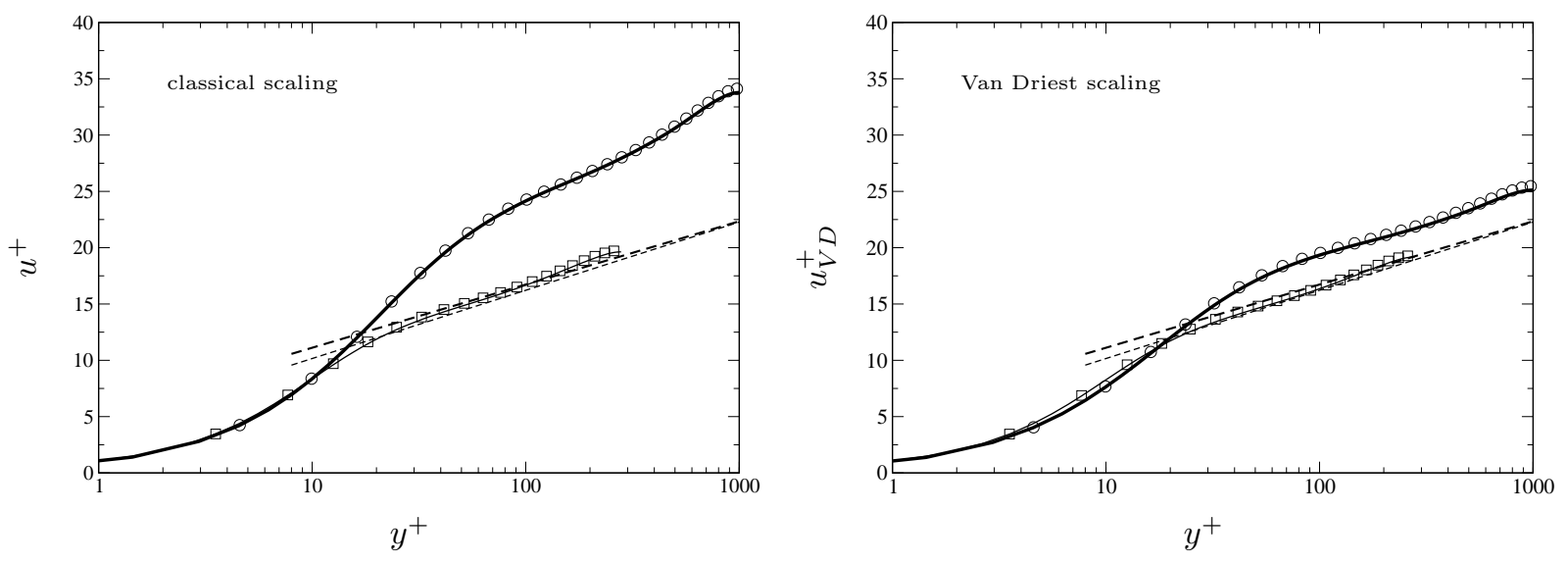

FIG. 20: Comparison between classical and Van Driest scaling for the mean streamwise velocity. $\square$ : case $A ; \quad \longrightarrow$ : case $B ; \quad O$ : case $C ; \quad-$ : case $D ; \quad-\mathbf{- ~}$ : standard law of the wall, $1 / \kappa \ln y^{+}+C$, with $\kappa=0.41$ and $C=5.5 ; \quad----$ : law of the wall advocated by Österlund $e t$ al.[53], $\kappa=0.38$ and $C=4.1$.

without chemical reactions. The collapse seems to be even better taking the values of $\kappa$ and $C$ proposed by Österlund et al.[53] but this should be confirmed with other experimental and numerical data.

\section{Finding a function for the total heat flux}

Finding a function similar to Eq. (25) for the total heat flux requires more attention when dealing with reacting flows. Indeed, the expression of $G$ depends on the energy conservation equation considered. For instance, if the total non chemical energy equation (4) is chosen, we end up with:

$$
\frac{d}{d y}\left(\overline{\rho v E}+\overline{q_{y}^{*}}-\overline{\tau_{y i} u_{i}}+\overline{p v}\right)=\overline{\dot{\omega}_{T}}+\bar{u} \mathcal{S}_{x}+\overline{\mathcal{Q}}
$$

where the RHS terms are neither null nor equal to a constant value. This is shown in Fig. 21 where the heat release is compared to the contribution of the two source terms. We clearly see that even if the kinetic energy source term $\bar{u} \mathcal{S}_{x}$ is negligible, the heat release $\overline{\dot{\omega}_{T}}$ is so strong near the wall that its contribution cannot be neglected. Moreover, since it is an unknown function of the distance to the wall, it cannot be integrated analytically. Hence, the derivation of the wall model cannot be achieved from the equation of total non chemical energy conservation.

Now considering the conservation of specific enthalpy, Eq. (22), one ends up with a more 

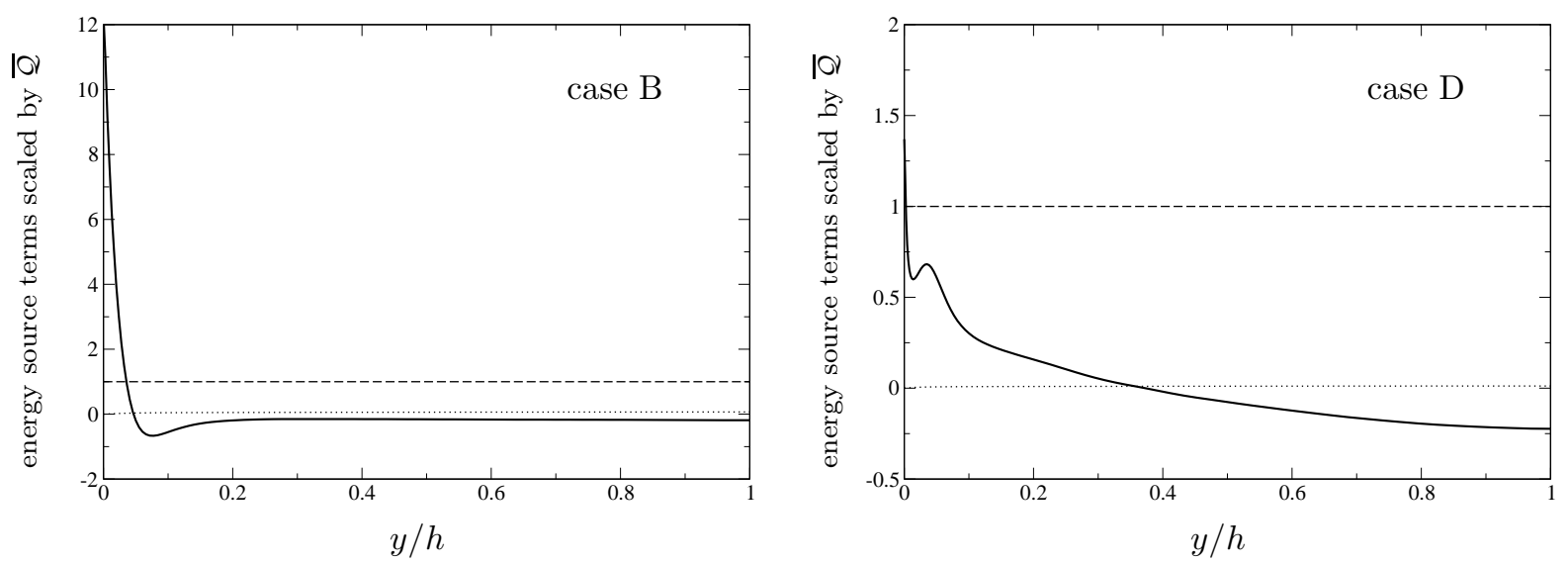

FIG. 21: RHS terms of Eq. 33 scaled by $\overline{\mathcal{Q}} . \quad \longrightarrow \quad$ : heat release, $\overline{\dot{\omega}_{T}} ; \quad \cdots \ldots \ldots$ : kinetic energy source term, $\bar{u} \mathcal{S}_{x} ; \quad----$ : volume energy source term, $\overline{\mathcal{Q}}$.

convenient expression because the heat release is no longer present as a source term and the following form is obtained:

$$
\frac{d \overline{q_{t o t}}}{d y} \approx \frac{d}{d y}\left(\bar{\rho} \widetilde{v^{\prime \prime} h_{s}^{\prime \prime}}+\bar{\rho} \sum_{k} \widetilde{v^{\prime \prime} Y_{k}^{\prime \prime}} \Delta h_{f, k}^{0}-\overline{\lambda \frac{d T}{d y}}+\bar{\rho} \sum_{k}\left\{h_{k} Y_{k} V_{k, y}\right\}\right)=\overline{\mathcal{Q}}
$$

where the power of pressure forces, $\overline{v d p / d y}$, and the viscous effects, $\overline{\tau_{i y} d u_{i} / d y}$ have been neglected in agreement with the conclusions of Sec. III C. Moreover, neglecting the viscous effects is supported by the fact that we want to develop a wall model for low Mach number configurations. The viscous effect contribution is displayed in Fig. 22 together with all the other terms to demonstrate that it has no influence on the assessment of the total heat flux at the wall. Indeed, the fluxes that are not null at the wall are the Fourier flux and the viscous effect flux but the latter one is only a few percents of the total heat flux at the wall. In addition, the 1-slope (due to the energy source term $\overline{\mathcal{Q}}$ ) is well recovered on the total heat flux which demonstrates the relevancy of the data sample. By analogy with the momentum source term $\mathcal{S}_{x}$, this none physical term can be set to zero in the context of wall modeling because it is a constant volume source term that does not change the behavior of other heat flux terms (except giving them a slope). In a real boundary layer $\overline{\mathcal{Q}}=0$ (the total heat flux is constant) and the term $G$ of the generic equation (25) is null. Note also that the species diffusion heat flux has no influence on the heat flux at the wall and quickly vanishes in the turbulent zone, $y / h>0.2$. For the case where the temperature gradient is significant (case D), the contribution of this term to the total heat flux is strongly lowered. The species 

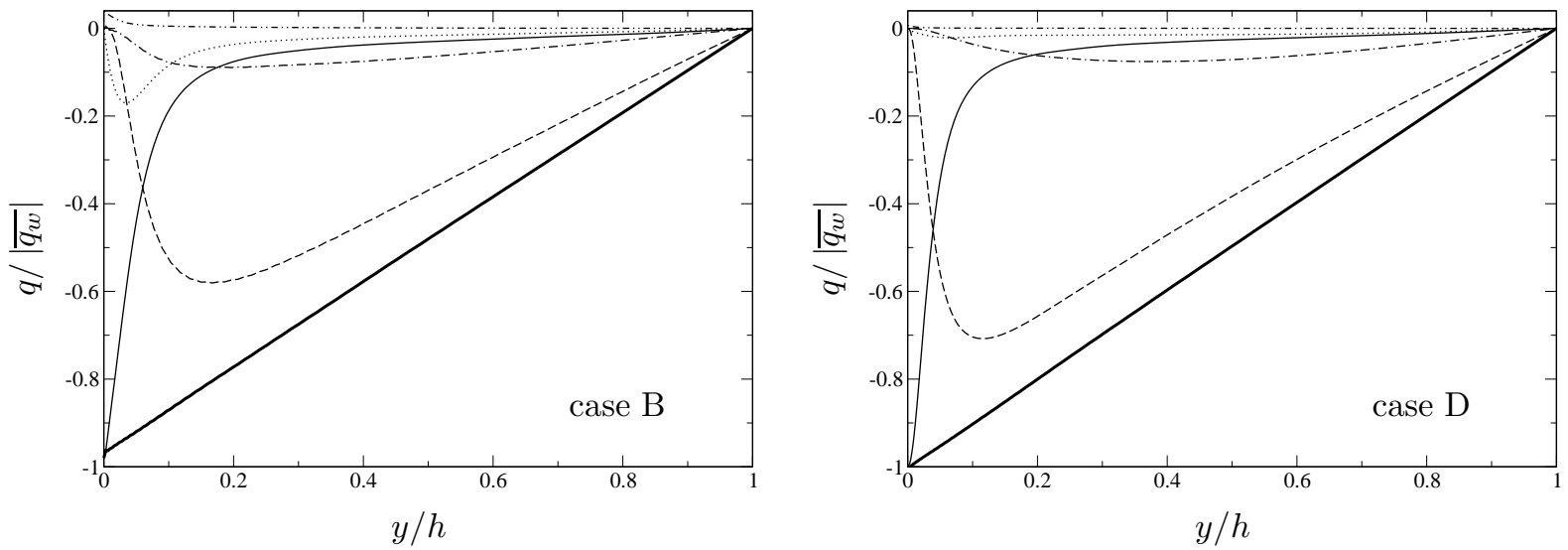

FIG. 22: Heat flux balance scaled by the modulus of the flux at the wall $\left|\overline{q_{w}}\right|$.

--- - : turbulent flux of sensible enthalpy, $\bar{\rho} \widetilde{v^{\prime \prime} h_{s}^{\prime \prime}} ; \quad-$ - - : turbulent flux of chemical enthalpy, $\bar{\rho} \sum_{k} \widetilde{v^{\prime \prime} Y_{k}^{\prime \prime}} \Delta h_{f, k}^{0}$; - Fourier heat flux, $-\overline{\lambda \frac{d T}{d y}} ; \quad$ …... : species diffusion flux, $\bar{\rho} \sum_{k}\left\{h_{k} Y_{k} V_{k, y}\right\} ; \quad-\cdots-$ : viscous effect integrated in the wall normal direction, $-\int \overline{\tau_{i y} \frac{d u_{i}}{d y}} d y$; $\quad$ : full total heat flux, $\overline{q_{t o t}}-$ $\int \overline{\tau_{i y} \frac{d u_{i}}{d y}} d y$.

diffusion heat flux can thus be neglected for developing the wall model. In the turbulent region, the dominant term is the turbulent flux of sensible enthalpy, but the turbulent flux of chemical enthalpy cannot be neglected. Hence, the total heat flux is approximated in the boundary layer by the relation:

$$
\overline{q_{t o t}} \approx \bar{\rho} \widetilde{v^{\prime \prime} h_{s}^{\prime \prime}}+\bar{\rho} \sum_{k} \widetilde{v^{\prime \prime} Y_{k}^{\prime \prime}} \Delta h_{f, k}^{0}-\overline{\lambda \frac{d T}{d y}}
$$

We clearly see in Fig. 23 that neglecting the species diffusion is a good assumption because the total heat flux is still well modeled in the turbulent region by only considering the turbulent flux of specific enthalpy (sum of the sensible and chemical enthalpies). Note also that the Fourier heat flux, that contains the turbulent term $\overline{\lambda^{\prime} d T^{\prime} / d y}$, is perfectly approximated by the strictly laminar Fourier flux $\bar{\lambda} d \bar{T} / d y$.

Two terms remains: the turbulent fluxes of sensible enthalpy and chemical enthalpy. The first one can be modeled with the classical approximation:

$$
\bar{\rho} \widetilde{v^{\prime \prime} h_{s}^{\prime \prime}} \approx \bar{\rho} \overline{C_{p}} \widetilde{v^{\prime \prime} T^{\prime \prime}} \approx-\lambda_{t} \frac{d \bar{T}}{d y}
$$

where $\lambda_{t}$ is the turbulent heat diffusion coefficient. Assuming a Reynolds analogy, $\lambda_{t}$ can be 

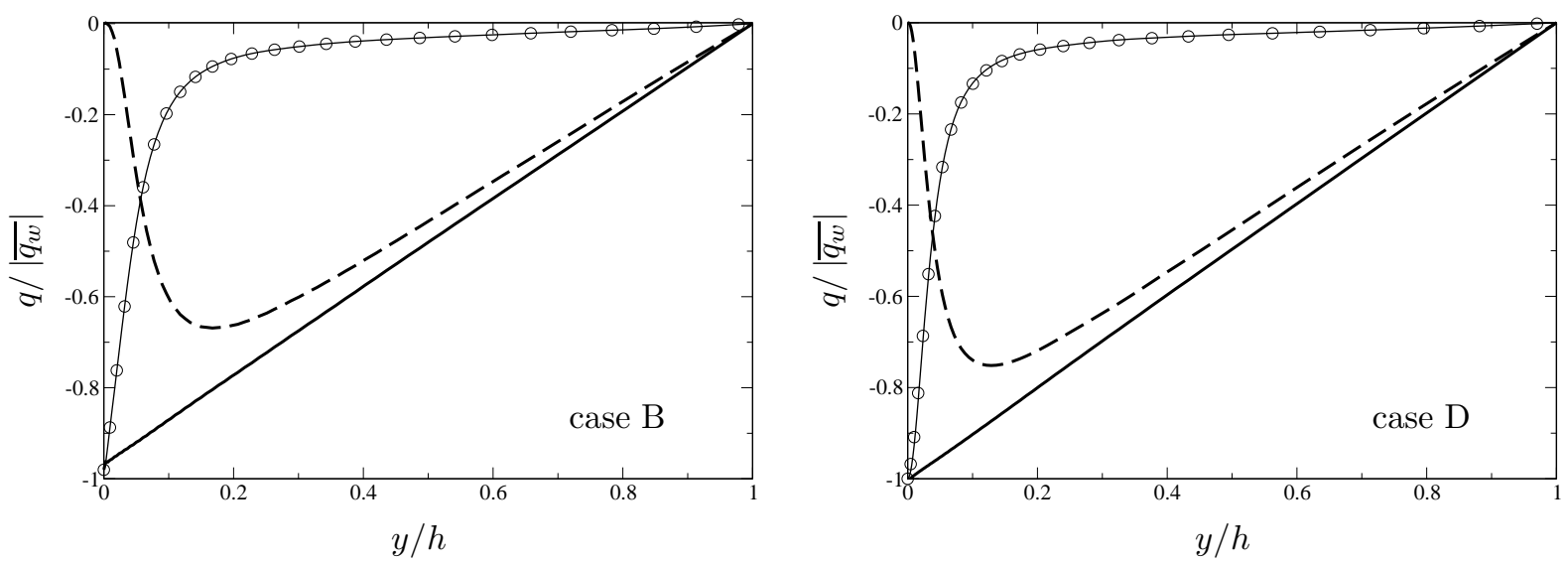

FIG. 23: Heat flux balance scaled by the modulus of the flux at the wall $\left|\overline{q_{w}}\right|$.

: Fourier flux, $-\overline{\lambda \frac{d T}{d y}} ; \quad$ - - - : turbulent flux of specific enthalpy, $\bar{\rho} \widetilde{v^{\prime \prime} h^{\prime \prime}}=\bar{\rho} \widetilde{v^{\prime \prime} h_{s}^{\prime \prime}}+\bar{\rho} \sum_{k} \widetilde{v^{\prime \prime} Y_{k}^{\prime \prime}} \Delta h_{f, k}^{0} ;$ — : full total heat flux, $\overline{q_{t o t}}-\int \overline{\tau_{i y} \frac{d u_{i}}{d y}} d y ; \quad \circ$ : approximation of the Fourier flux, $-\bar{\lambda} \frac{d \bar{T}}{d y}$.

expressed as:

$$
\lambda_{t}=\frac{\mu_{t} \overline{C_{p}}}{P r_{t}}
$$

where $P r_{t}$ is the turbulent Prandtl number, usually taken to a constant value. Figure 24 shows the evolution of the turbulent Prandtl number computed in the simulations as:

$$
\operatorname{Pr}_{t}=\frac{\widetilde{u^{\prime \prime} v^{\prime \prime}} d \bar{T} / d y}{\widetilde{v^{\prime \prime} T^{\prime \prime}} d \bar{u} / d y}
$$

and suggests that $\operatorname{Pr}_{t}=0.7$ for the current set of simulations which is an acceptable value[80]. This figure also reveals that chemical reactions and strong temperature gradient do not influence the turbulent Prandtl number behavior because the results are similar for case A, B, C and D. Finally, we propose the following expression to model the turbulent flux of sensible enthalpy:

$$
\bar{\rho} \widetilde{v^{\prime \prime} h_{s}^{\prime \prime}} \approx-\frac{\mu_{t} \overline{C_{p}}}{P r_{t}} \frac{d \bar{T}}{d y}
$$

This model is tested with the database of cases $\mathrm{B}$ and $\mathrm{D}$, and the results are reported in Fig. 25 that shows a good agreement for the first approximation, $\bar{\rho} \widetilde{v^{\prime \prime} h_{s}^{\prime \prime}} \approx \bar{\rho} \overline{C_{p}} \widetilde{v^{\prime \prime} T^{\prime \prime}}$. Moreover, the model with $\operatorname{Pr}_{t}=0.7$ and the value of $\mu_{t}$ taken from the simulations (i.e. $\left.\mu_{t}=-\bar{\rho} \widetilde{u^{\prime \prime} v^{\prime \prime}} /(d \bar{u} / d y)\right)$ gives a very good prediction for the turbulent flux of sensible enthalpy attesting the quality of this model. Note that the value of $\mu_{t}$ could have also been modeled with the Prandtl mixing-length model described above but the goal here is to discuss the model with a constant value of $P r_{t}$ assuming that $\mu_{t}$ is perfectly approximated. 


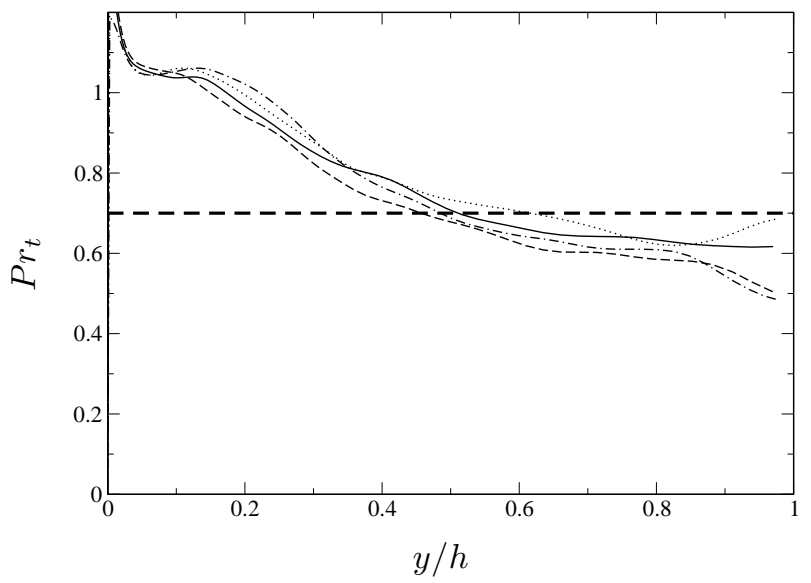

FIG. 24: Turbulent Prandtl number. $\quad-.-$ : case A; $\quad$....... : case B; $\quad----$ : case C; : case $\mathrm{D} ; \quad \mathbf{-}-\mathbf{-}: \mathrm{Pr}_{t}=0.7$.
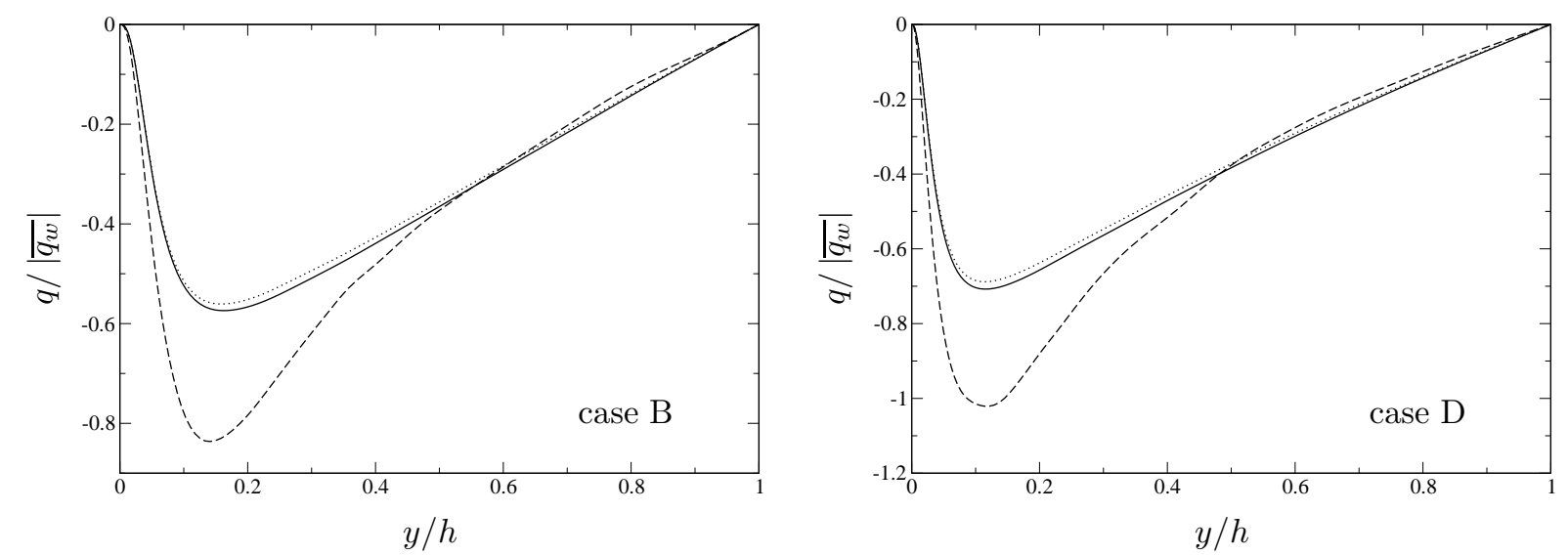

FIG. 25: A priori test for the turbulent flux of sensible enthalpy. _ $\quad$ : simulation, turbulent flux of sensible enthalpy, $\bar{\rho} \widetilde{v^{\prime \prime} h_{s}^{\prime \prime}} ; \quad \cdots \cdots . .$. : simulation, first approximation, $\bar{\rho} \overline{C_{p}} \widetilde{v^{\prime \prime} T^{\prime \prime}} ; \quad$ - - - : : model, $-\frac{\mu_{t} \overline{C_{p}}}{P r_{t}} \frac{d \bar{T}}{d y}$.

Concerning the turbulent flux of chemical enthalpy, one makes use of a gradient type model for species diffusion closed with a turbulent Schmidt number $S c_{t, k}$ for each $k$-species. We recall that the chemical equilibrium assumption for the mixture composition has been verified in Sec. III A which allows to propose the following model for the turbulent diffusion of each $k$-species:

$$
\bar{\rho} \widetilde{v^{\prime \prime} Y_{k}^{\prime \prime}} \approx-\frac{\mu_{t}}{S c_{t, k}} \frac{W_{k}}{\bar{W}} \frac{d \overline{X_{k}}}{d y} \approx-\left.\frac{\mu_{t}}{S c_{t, k}} \frac{W_{k}}{\bar{W}} \frac{d \overline{X_{k}}}{d \bar{T}}\right|_{e q} \frac{d \bar{T}}{d y}
$$


Summing over all the species finally gives the model for turbulent flux of chemical enthalpy:

$$
\bar{\rho} \sum_{k} \widetilde{v^{\prime \prime} Y_{k}^{\prime \prime}} \Delta h_{f, k}^{0} \approx-\left.\sum_{k} \frac{\mu_{t}}{S c_{t, k}} \frac{W_{k}}{\bar{W}} \frac{d \overline{X_{k}}}{d \bar{T}}\right|_{e q} \frac{d \bar{T}}{d y} \Delta h_{f, k}^{0}
$$

In order to determine which values of the turbulent Schmidt numbers could be retained for the final model, they have been computed in the simulations as:

$$
S c_{t, k}=\frac{\widetilde{u^{\prime \prime} v^{\prime \prime}} d \overline{X_{k}} / d y}{\widetilde{v^{\prime \prime} X_{k}^{\prime \prime}} d \bar{u} / d y}
$$

Averaging over all the species, the mean turbulent Schmidt number and its standard deviation are obtained and shown in Fig. 26. The same treatment is also done for the turbulent Lewis numbers, $L e_{t, k}=S c_{t, k} / P r_{t}$. A first result visible in this figure is that the standard deviation of the turbulent Schmidt and Lewis numbers for all the species remain weak. This is convenient because we can assume from this result that $S c_{t, k}$ and $L e_{t, k}$ are not dependent on the species which means that a unique value can be imposed. Moreover, it is verified that the classical approximation consisting in taking the turbulent Lewis numbers equal to unity is a very good model. This illustrates that the turbulent diffusion of the species and of the temperature behave in a similar way. The wall model is then built with the assumption $S c_{t, k} \approx P r_{t}$. Hence, Eq. (41) is tested in Fig. 27 taking the profile of temperature from the simultions and $S c_{t, k}=0.7$. The turbulent dynamic viscosity $\mu_{t}$ is also taken from the simulations so that we can check the quality of this model without including the effects of the Prandtl mixing-length model. Finally, Eq. (41) appears to be an efficient model.

Now that we have determined the appropriated models for the turbulent contributions of the total heat flux, we can follow the derivation of the wall model by replacing Eq. (39) and Eq. (41) into Eq. (35). Moreover, in a modeling context, the laminar Fourier heat flux can be neglected because the first off-wall point is in the fully turbulent region, and $\overline{q_{t o t}}$ can be replaced by $\overline{q_{w}}$ because the total heat flux is constant when $\overline{\mathcal{Q}}=0$. All these manipulations leads to the following model for the wall heat flux:

$$
\overline{q_{w}} \approx-\mu_{t}\left(\frac{\overline{C_{p}}}{P r_{t}}+\left.\frac{1}{S c_{t, k}} \sum_{k} \frac{W_{k}}{\bar{W}} \frac{d \overline{X_{k}}}{d \bar{T}}\right|_{e q} \Delta h_{f, k}^{0}\right) \frac{d \bar{T}}{d y}
$$

\section{Derivation of the coupled model}



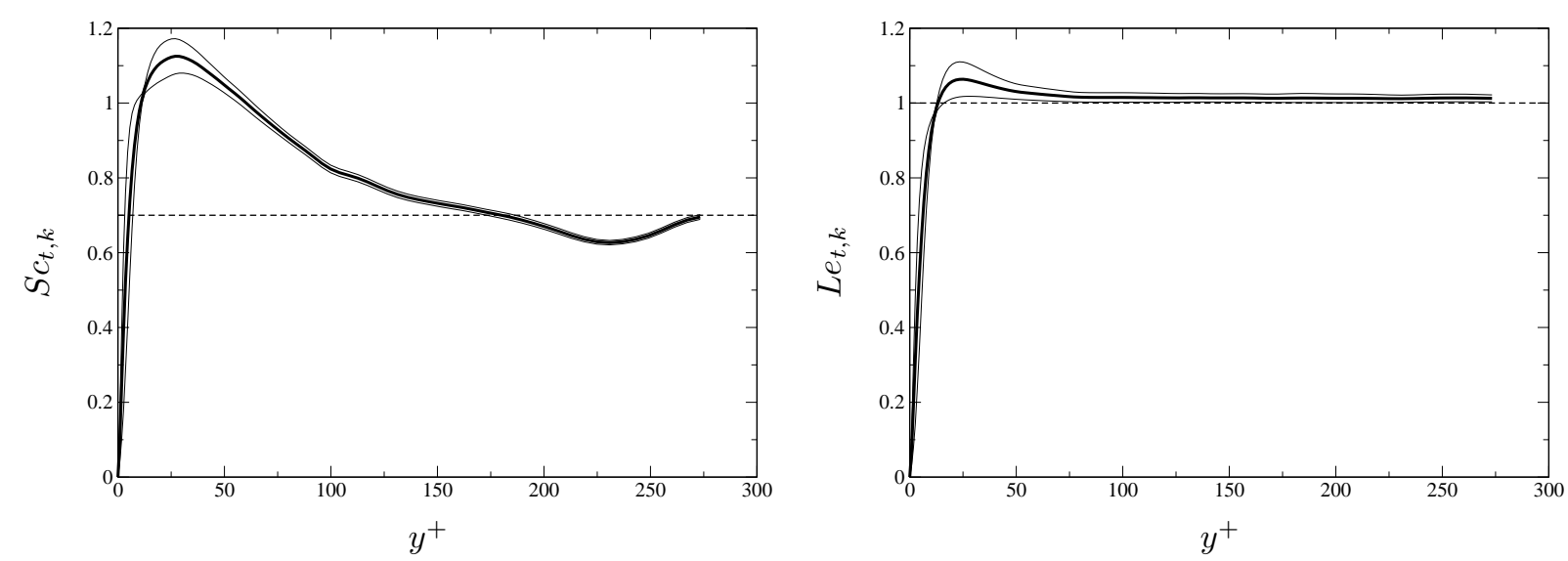

FIG. 26: Turbulent Schmidt and Lewis numbers of case B (results for case D are similar). — : averaged value of all the species; — $\quad$ : averaged value \pm standard deviation; --- : models proposed, $S c_{t, k}=0.7$ and $L e_{t, k}=1$.
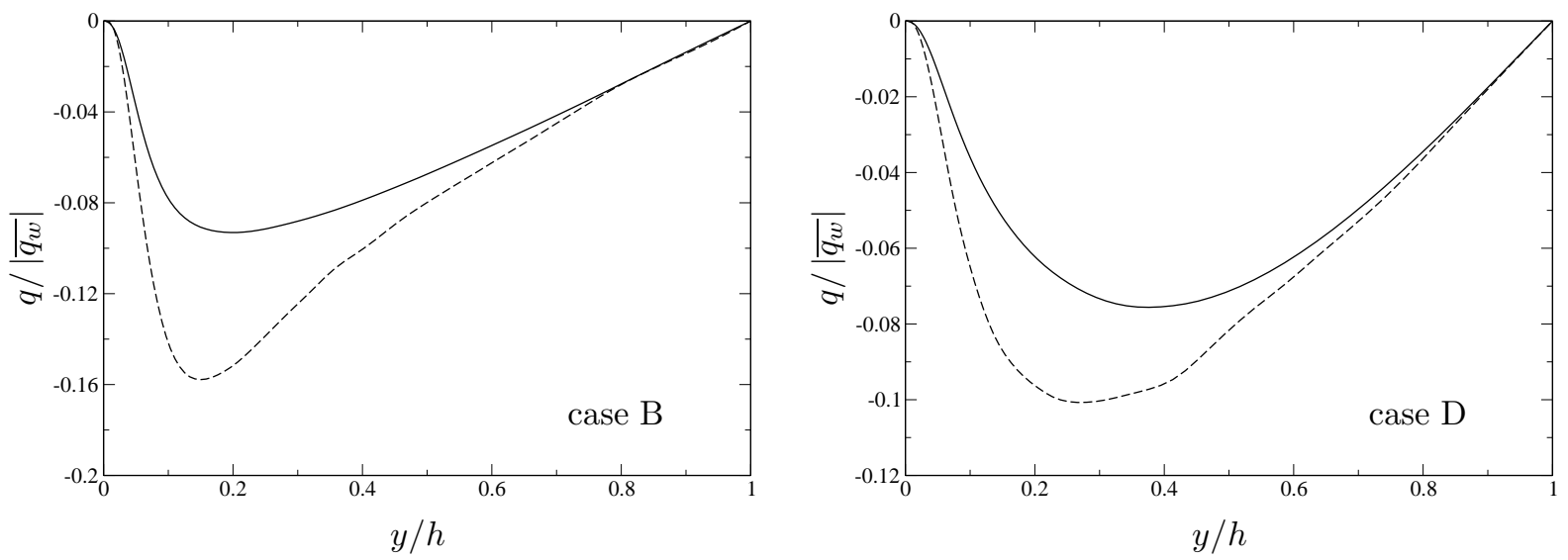

FIG. 27: A priori test for the model of turbulent flux of chemical enthalpy. : simulation, turbulent flux of chemical enthalpy, $\bar{\rho} \sum_{k} \widetilde{v^{\prime \prime} Y_{k}^{\prime \prime}} \Delta h_{f, k}^{0} ; \quad----:$ model, $-\left.\sum_{k} \frac{\mu_{t}}{S c_{t, k}} \frac{W_{k}}{\bar{W}} \frac{d \overline{X_{k}}}{d \bar{T}}\right|_{e q} \frac{d \bar{T}}{d y} \Delta h_{f, k}^{0}$.

The first part of the wall model derivation has led to an expression for $\overline{\tau_{w}}$, the mean shear stress at the wall Eq. (30), and for $\overline{q_{w}}$, the mean wall heat flux Eq. (43). These two equations constitute a system that we will manipulate in order to find the equations for the velocity and the temperature in wall units.

We start by computing the ratio $\overline{q_{w}} / \overline{\tau_{w}}$ which gives the following expression for the 

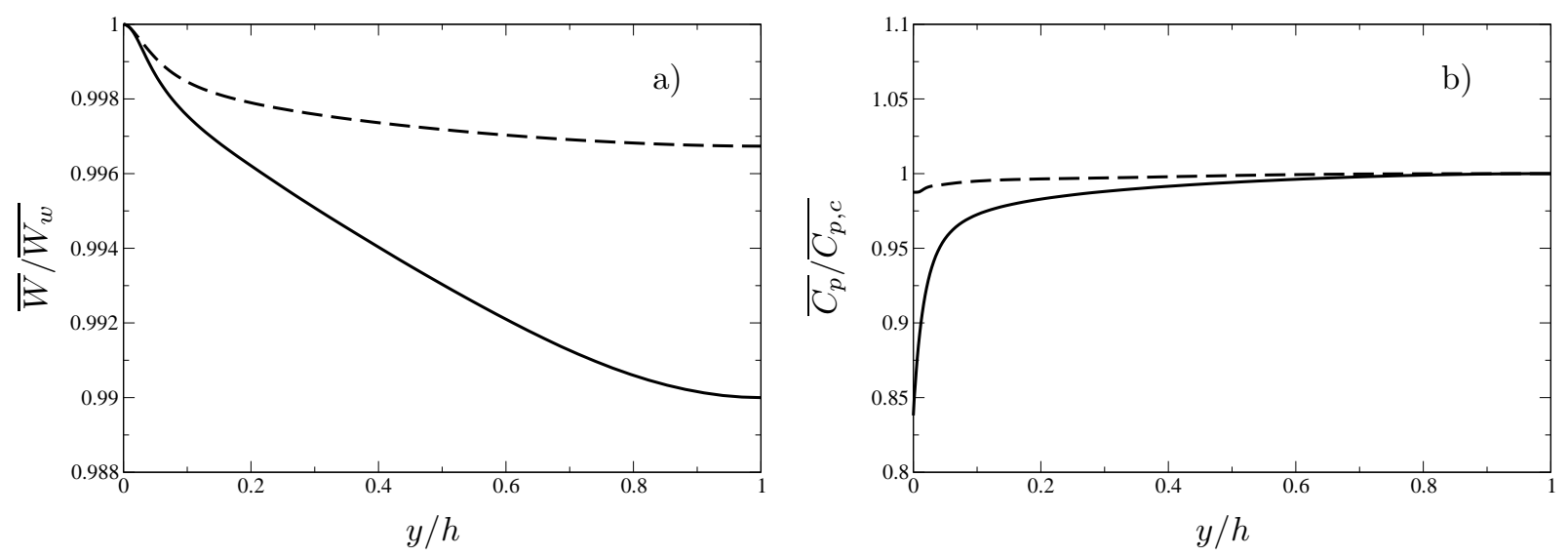

FIG. 28: a) Mean molecular weight profile scaled by its value at the wall. b) Mean heat capacity at constant pressure scaled by its value at the centerline. - - - : case B; — : case D.

temperature gradient:

$$
\frac{d \bar{T}}{d y}=-\frac{\overline{q_{w}}}{\overline{\tau_{w}}\left(\frac{\overline{C_{p}}}{P r_{t}}+\left.\frac{1}{S c_{t, k}} \sum_{k} \frac{W_{k}}{\bar{W}} \frac{d \overline{X_{k}}}{d \bar{T}}\right|_{e q} \Delta h_{f, k}^{0}\right)} \frac{d \bar{u}}{d y}
$$

Integrating this relation in the wall normal direction and assuming that $\overline{C_{p}}$ and $\bar{W}$ do not vary too much in the boundary layer $\left(\overline{C_{p}(y)}=C_{p}\right.$ and $\overline{W(y)}=W$, justified by Fig. 28) leads to an equation of the type:

$$
\frac{\bar{T}}{T_{w}}=D-\alpha u^{+}
$$

where $D$ is an integration constant, and:

$$
\alpha=\frac{C_{p} B_{q}}{\frac{C_{p}}{P r_{t}}+\left.\frac{1}{S c_{t, k}} \sum_{k} \frac{W_{k}}{W} \frac{d \overline{X_{k}}}{d \bar{T}}\right|_{e q} \Delta h_{f, k}^{0}}
$$

and:

$$
B_{q}=\frac{T_{\tau}}{T_{w}}=\frac{\overline{q_{w}}}{\overline{\rho_{w}} C_{p} u_{\tau} T_{w}}
$$

Making use of the wall unit scaling for the temperature gives the generic expression for $T^{+}$:

$$
T^{+}=\frac{1-D}{B_{q}}+\frac{\alpha}{B_{q}} u^{+}
$$

where the integration constant $D$ still has to be determined.

Because the variation of the ratio $\bar{W} / \overline{W_{w}}$ is negligible in the boundary layer (Fig. 28 shows that even for the case of significant temperature gradient the variation is less than 
$1 \%$ ), we can use the equation of state for perfect gas and write $\bar{\rho} / \rho_{w} \approx T_{w} / \bar{T}$. Thanks to this relation and Eq. (45), the Van Driest transformation Eq. (32) can be integrated as:

$$
\int_{0}^{u^{+}}\left(\frac{T_{w}}{\bar{T}}\right)^{1 / 2} d u^{+}=\int_{0}^{u^{+}}\left(\frac{1}{D-\alpha u^{+}}\right)^{1 / 2} d u^{+}=\frac{1}{\kappa} \ln y^{+}+C
$$

which finally gives an equation for $u^{+}$:

$$
\frac{2}{\alpha}\left(\sqrt{D}-\sqrt{D-\alpha u^{+}}\right)=\frac{1}{\kappa} \ln y^{+}+C
$$

We now have the two equations of the coupled wall model, Eq. (50) and Eq. (48), but we still have to determine the two constants $C$ and $D$ in order to complete the development of the wall model. Concerning the first one, we have seen in Fig. 20 that the classical value $C=5.5$ for wall-bounded flow can be assumed (the value $C=5.2$ being reserved for external boundary layers), associated with $\kappa=0.41$. Note that the values advocated by Österlund et al. [53], $\kappa=0.38$ and $C=4.1$, could have been also retained.

The constant $D$ is determined by imposing the wall model to recover the classical logarithmic formulations for velocity and temperature for non-reacting isothermal flows. Hence, $D$ must satisfy two constraints:

- a) when $B_{q} \rightarrow 0$ (i.e. the flow tends to be isothermal), one must have $D \rightarrow 1$ to recover the classical logarithmic law for velocity;

- b) when $B_{q} \rightarrow 0$, Eq. (48) must be bounded. For instance, this can be verified if $(1-D) / B_{q}$ is a constant that does not depend on $B_{q}$.

Because in a turbulent boundary layer the temperature profile is sensitive to the molecular Prandtl number[77], one decides to pose:

$$
\frac{1-D}{B_{q}}=K(P r)
$$

where $K(P r)$ is a constant that only depends on the molecular Prandtl number. In this way, the two constraints for $D$ are satisfied. Hence, when $B_{q} \rightarrow 0$ (isothermal case) and $\alpha \rightarrow \operatorname{Pr}_{t} B_{q}$ (no chemistry) the two equations, Eq. (50) and Eq. (48), tends to the classical logarithmic formulations $\left(u^{+}=1 / \kappa \ln y^{+}+C\right.$ and $\left.T^{+}=P r_{t} / \kappa \ln y^{+}+\operatorname{constant}(\operatorname{Pr})\right)$. To go further in the determination of the $K(P r)$ constant, one chooses to make use of the Kader's function[8] as the reference equation for temperature profile because it takes into account 
the effect of the molecular Prandtl number in its formulation (for $6 \times 10^{-3}<\operatorname{Pr}<40 \times 10^{3}$ ). When the laminar part of the Kader's function tends to zero, the turbulent part remains and we have:

$$
T_{K}^{+}=2.12 \ln y^{+}+\beta(P r)
$$

where $\beta(P r)$ is a function of the molecular Prandtl number defined as:

$$
\beta(\operatorname{Pr})=\left(3.85 \operatorname{Pr}^{1 / 3}-1.3\right)^{2}+2.12 \ln \operatorname{Pr}
$$

One arbitrarily decides to determine the $K(P r)$ constant by minimizing the square difference between Eq.(52) and Eq.(48) (i.e. $\left.\left(T^{+}-T_{K}^{+}\right)^{2}\right)$ in the limit $B_{q} \rightarrow 0$ and $\alpha \rightarrow P r_{t} B_{q}$, for $100<y^{+}<200$. This leads to an analytical expression for $K(\operatorname{Pr})$ :

$$
K(P r)=\beta(P r)-P r_{t} C+\left(\frac{P r_{t}}{\kappa}-2.12\right)(1-2 \ln (20))
$$

Hence, the wall model is able to take into account the effects of the molecular Prandtl number, the strong temperature gradients, and the chemical reactions while being consistent with the classical logarithmic formulations for velocity and temperature.

Now that all the terms of the wall model are defined one can proceed to the evaluation of its efficiency performing a priori tests.

\section{A priori tests}

Knowing a set of outer conditions $\left(u_{\text {out }}, T_{\text {out }}, Y_{k, \text { out }}\right)$ at the wall distance $y_{\text {out }}$, and the temperature at the wall $T_{w}$ it is possible to invert the system of equations, Eq. (48) and Eq. (50), to assess the wall fluxes. In order to test the efficiency of this coupled wall model several sets of outer conditions have been built from the mean profiles of the present simulations (the mean velocity, mean temperature and mean mass fraction profiles are used to generate $u_{\text {out }}, T_{\text {out }}$, and $Y_{k, \text { out }}$, respectively). The wall fluxes resulting from the inversion of the coupled wall model can then be confronted to their exact values taken from the simulations. The parameters used for the inversion are summed up in table IX where $R$ is the perfect gas constant, $\left.W_{w}\right|_{e q}=\left.\sum_{k} X_{k, w}\right|_{e q} W_{k}$ is the molecular weight of the mixture at the wall determined with the equilibrium assumption, $\left.C_{p, w}\right|_{e q}=\left.\sum_{k} C_{p, k} Y_{k, w}\right|_{e q}$ is the heat capacity at constant pressure evaluated at the wall from the wall temperature (which is a known value) and the equilibrium concentration profiles, and $W_{\text {out }}=\sum_{k} X_{k, \text { out }} W_{k}$ the molecular weight at the outer condition. Note that because the pressure is constant in the 

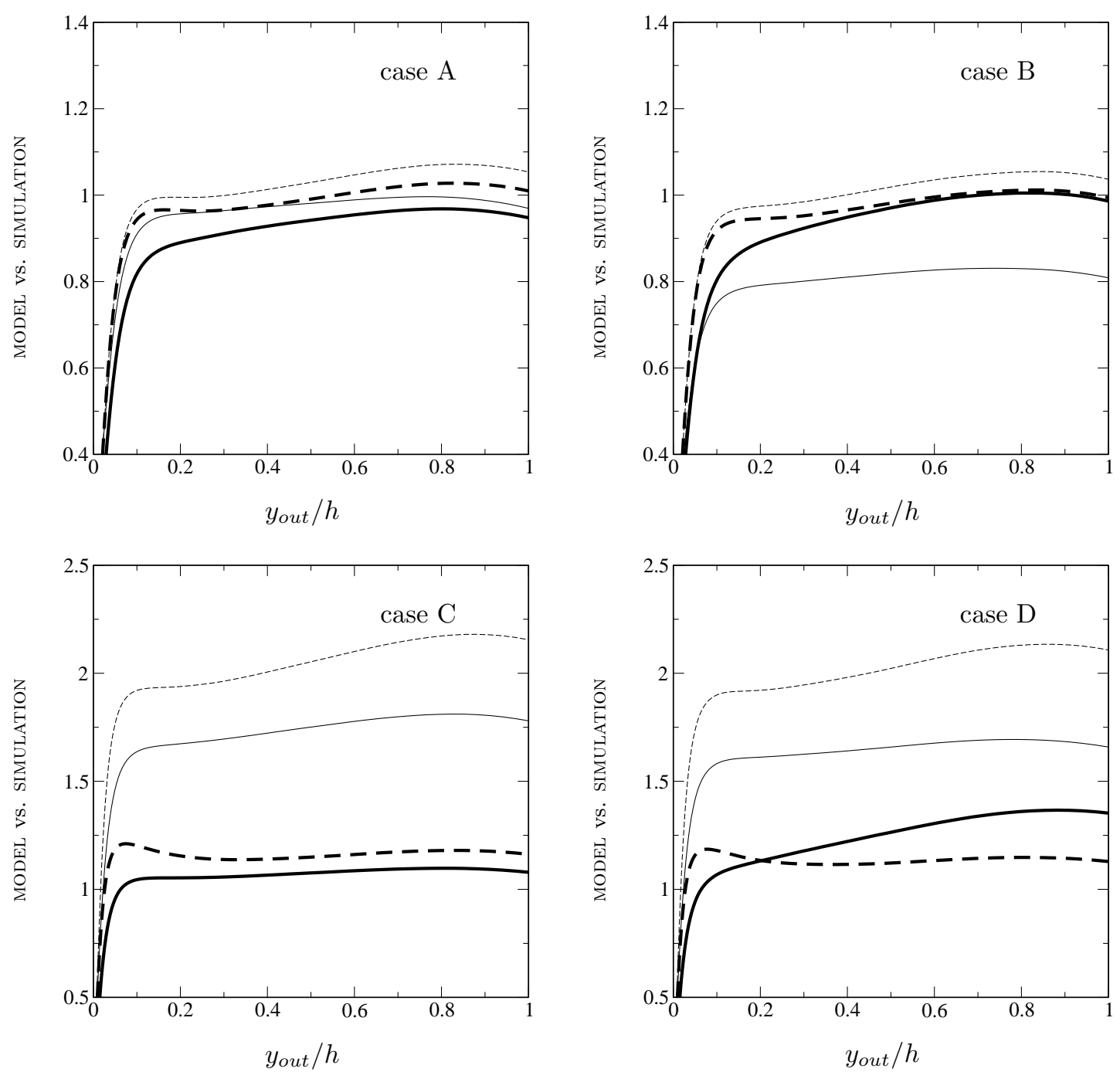

FIG. 29: Wall heat flux and viscous stress scaled by their exact counterparts from the DNS/LES. - - - : $\tau_{w}$ from the new wall model; — $: q_{w}$ from the new wall model; $----: \tau_{w}$ from the standard law-of-the-wall, $u^{+}=1 / \kappa \ln y^{+}+C ; \quad \longrightarrow: q_{w}$ from the Kader correlation[8], Eq. (52).

boundary layer, the outer pressure $p_{\text {out }}$ is used to determine the wall density. Results are shown in Fig. 29. In these plots, the predicted wall shear stress, $\tau_{w}^{\text {model }}$, and wall heat flux, $q_{w}^{\text {model }}$, are scaled by their exact DNS/LES counterparts. The values given by the standard wall function for mean velocity and by the Kader's correlation for temperature[8] are also plotted to illustrate the efficiency of the new model. Note that in Fig. 29 the determination of $q_{w}$ from the Kader function, Eq. (52), is done by determining $y^{+}$thanks to the classical law-of-the-wall for velocity.

For all the cases, the coupled wall model is more efficient than standard functions: the 
TABLE IX: Set of variables retained for the inversion of the law-of-the-wall Eq. (48) and Eq. (50).

\begin{tabular}{cccccccc}
\hline \hline$\kappa$ & $C$ & $K(P r)$ & $P r_{t}$ & $S c_{t, k}$ & $\overline{\rho_{w}}$ & $C_{p}$ & $W$ \\
\hline 0.41 & 5.5 & -0.5 & 0.7 & 0.7 & $\left.T_{w} W_{w}\right|_{\text {eq }} / R p_{\text {out }}$ & $\left.C_{p, w}\right|_{\text {eq }}$ & $W_{\text {out }}$ \\
\hline \hline
\end{tabular}

new model exhibits a large plateau for $y_{\text {out }}>0.2 h$ where the error is a few percents (between $5 \%$ and $20 \%$, say). Of course profiles vanish for $y_{\text {out }}<0.2 h$ because the wall model is not expected to hold in the viscous sublayer. In case A, the effects of variable density and chemistry are not present which explains a good collapse of both the new and standard models. In the case of multicomponent reacting flow with smooth temperature gradient (case B), the model clearly improves the prediction which shows that the chemical part of the model behaves well: with the new wall model, the error on the wall heat flux prediction is damped to $5 \%$ whereas the error is around $20 \%$ for the standard model.

Concerning the strong temperature gradient cases $\mathrm{C}$ and $\mathrm{D}$, the standard functions commit an error of $100 \%$ and $60 \%$ on the wall shear stress and heat flux respectively. These predictions are clearly improved by the new wall model developed herein which works pretty well without chemistry and strong temperature variations (in case C, errors are lowered to $15 \%$ for the wall shear stress and $10 \%$ for the wall heat flux) and shows a moderate discrepancy when chemical reactions are considered (case D).

The current model has been developed under the assumption of a flow at infinite Reynolds number. The DNS/LES are at moderate Reynolds number which could explain the little discrepancies observed in these a priori tests. Moreover, one recall that no specific tuning of the free coefficients has been done because the aim of this study is to obtain a law-of-thewall with appropriate dependancies and scaling between the wall unknowns and the outer variables. For instance, the error in the prediction of the wall heat flux can be lowered by $8 \%$ for case D, taking $\operatorname{Pr}_{t}=0.65$ (which would fit better the DNS data than $\operatorname{Pr}_{t}=0.7$ ), and the values advocated by Österlund et al.[53] for $C$ and $\kappa$.

Hence, the velocity/temperature/chemistry coupled law-of-the-wall developed in this study appears to behave well with reacting flows at low Mach number, with or without temperature gradient which opens a wide range of numerical investigations (by RANS calculations) for industrial applications in such a flow configuration. 


\section{Adaptation of the wall model to the standard $k-\epsilon$ model for RANS calculations}

When wall functions are used in a numerical solver, one needs to prescribe a model for each transported variable. Thus wall functions have to be provided not only for velocity and energy variables but also for any turbulence variables simulated. The most implemented turbulence model for RANS calculations is probably the $k-\epsilon$ one[81] that transports the turbulence energy, $k=\widetilde{u_{i}^{\prime \prime} u_{i}^{\prime \prime}} / 2$, and its dissipation rate, $\epsilon$. This model is based on a linear constitutive law of the type:

$$
\widetilde{u_{i}^{\prime \prime} u_{j}^{\prime \prime}}=-2 \nu_{t} S_{i j}+\frac{2}{3} k \delta_{i j}
$$

where $S_{i j}=\left(\partial u_{i} / \partial x_{j}+\partial u_{j} / \partial x_{i}\right) / 2$, and $\nu_{t}=C_{\mu} k^{2} / \epsilon$. The turbulence production, $\mathrm{P}$, is then expresses as $P=2 \nu_{t} S_{i j} S_{i j}$.

Hence, for duct flows or external boundary layers, the expression of turbulence production is reduced to:

$$
P=-\widetilde{u^{\prime \prime} v^{\prime \prime}} \frac{d \bar{u}}{d y}
$$

which is modeled in the $\mathrm{k}-\epsilon$ model by:

$$
P=C_{\mu} \frac{k^{2}}{\epsilon}\left(\frac{d \bar{u}}{d y}\right)^{2} .
$$

Moreover, identifying Eq. (56) and Eq. (57), and making use of the classical assumption that production and dissipation of turbulence energy are in balance in the near wall region, $P=\epsilon$, we find the classical result $C_{\mu}={\widetilde{u^{\prime \prime} v^{\prime \prime}}}^{2} / k^{2}$. Because the experiments show that in the inertial logarithmic region of a boundary layer, $-\widetilde{u^{\prime \prime} v^{\prime \prime}} / k=0.3$, the constant of the model is set to $C_{\mu}=0.09$. As shown in Fig. 30, the present simulations also support the use of this value for the reacting compressible turbulent boundary layer. Finally, we find the standard relation between the turbulent shear stress and the turbulence energy:

$$
C_{\mu}^{1 / 2} k=-\widetilde{u^{\prime \prime} v^{\prime \prime}}
$$

We have seen that the laminar shear stress vanishes in the turbulent region and for this reason we can assume that the shear stress is constant in the turbulent region and thus equals the wall shear stress, $\tau_{w}$. Rearranging Eq. (27) we find that:

$$
\overline{\rho_{w}} u_{\tau}^{2} \approx-\bar{\rho} \widetilde{u^{\prime \prime} v^{\prime \prime}}
$$

and combining this equation with Eq. (58) we find the final expression for $k$ :

$$
k=\frac{\overline{\rho_{w}}}{\bar{\rho}} \frac{u_{\tau}^{2}}{C_{\mu}^{1 / 2}} .
$$




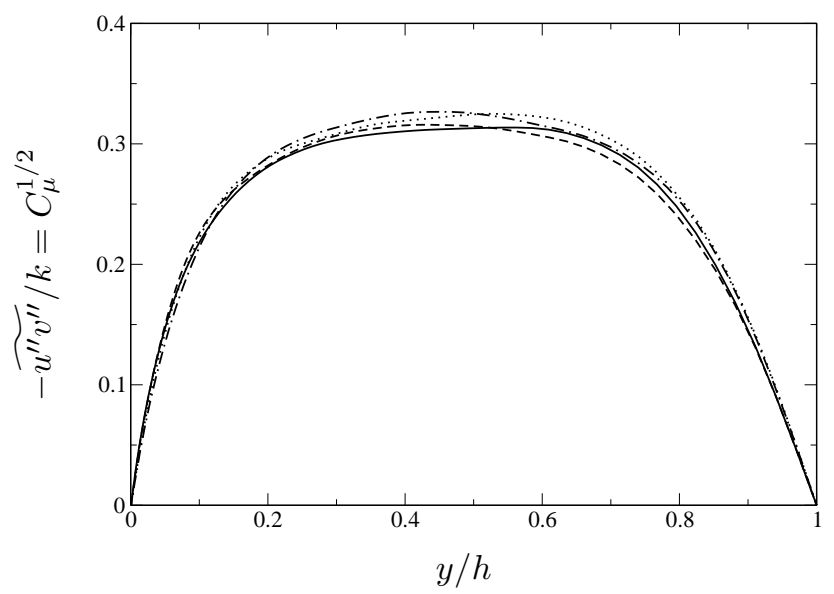

FIG. 30: Evaluation of $C_{\mu}^{1 / 2}$ in the present simulations. $\quad-.-$ : case $\mathrm{A} ; \quad \cdots \cdots . .$. : case B; - - - : : case C; — : case D.

Regarding the dissipation rate, because equilibrium between production and dissipation is assumed, we find the the same expression as Eq. (56):

$$
\epsilon=-\widetilde{u^{\prime \prime} v^{\prime \prime}} \frac{d \bar{u}}{d y}
$$

and the use of Eq. (59) allows to go further:

$$
\epsilon=\frac{\bar{\rho}}{\overline{\rho_{w}}} u_{\tau}^{2} \frac{d \bar{u}}{d y}
$$

where $d \bar{u} / d y$ can be expressed by rearranging Eq. (30):

$$
\frac{d \bar{u}}{d y}=\left(\frac{\overline{\rho_{w}}}{\bar{\rho}}\right)^{1 / 2} \frac{u_{\tau}}{\kappa y}
$$

which finally gives the expression for $\epsilon$ :

$$
\epsilon=\left(\frac{\bar{\rho}}{\overline{\rho_{w}}}\right)^{1 / 2} \frac{u_{\tau}^{3}}{\kappa y}
$$

Hence, Eq. (60) and Eq. (64) give the expressions to prescribe for $k$ and $\epsilon$ in the turbulent region. Compressibility (arising from a significant temperature gradient) and chemistry effects appear in the term $u_{\tau}$, determined by the resolution of the coupled system formed by Eq. (48) and Eq. (50). The ratio $\overline{\rho_{w}} / \bar{\rho}$ also translates the strong temperature gradient effect on $k$ and $\epsilon$. Note that when the temperature variation tends to zero, this ratio tends to one and the classical expressions, $k=u_{\tau}^{2} / C_{\mu}^{1 / 2}$ and $\epsilon=u_{\tau}^{3} /(\kappa y)$, are recovered. 
The integration of these two expressions into a numerical solver depends of course of the numerical method retained but we remind that Eq. (60) and Eq. (64) are only valid in the logarithmic region. For a cell-centered solver, it could be interesting to combine these equations with the method proposed by Craft et al. [50,52] to account for a correct behavior of $k$ and $\epsilon$ till the wall, and so in the laminar region.

\section{CONCLUSIONS}

The current paper presents a set of DNS and wall-resolved LES of periodic plane channel flow that constitutes a reference database for analyzing mean behavior of wall turbulence in the case of multicomponent reacting flows at low Mach number, with or without compressibility effect (the compressibility being due to a strong temperature gradient).

The study has revealed that the classical assumptions for boundary layer flows are still valid for multicomponent reacting flows, among them: the efficiency of the Boussinesq assumption combined with the Prandtl mixing-length for modeling the turbulent shear stress; the validity of the Van Driest transformation up to a temperature ratio of 3; and the efficiency of the Reynolds analogy for modeling the turbulent flux of sensible enthalpy. Moreover, it has been demonstrated that the power of pressure forces and the viscous effect terms could be neglected in the process of energy conservation.

This work has also presented some new findings concerning the multicomponent part of the Navier-Stokes equations for turbulent boundary layer flows. First, it appears that the composition of the mixture is at the equilibrium state which strongly simplifies the modeling. Second, the molecular species diffusion is negligible in the turbulent region. Third, a model for the turbulent flux of chemical enthalpy has been proposed and successfully validated. In addition, the turbulent Prandtl number appears to be insensitive to the chemical reactions and the strong temperature gradient. The present data also confirm that taking the turbulent Lewis number to unity is a good approximation which implies to take $S c_{k, t}=P r_{t}$. Note finally that it has also been shown that the Dufour part of the molecular heat flux could be neglected for such cases of study.

Simplifying the Navier-Stokes equations and replacing the turbulent terms by their model conterpart, a new law-of-the-wall useable in RANS computations is proposed to estimate the wall shear stress as well as the wall heat flux from the knowledge of a set 
of outer/wall conditions. This formulation stands for the general case of multicomponent reacting compressible turbulent flows and degrades in the classical formulations when the temperature gradient tends to zero and the chemistry effects are null. This law-of-the-wall also recognizes that the energy and momentum equations are coupled for large temperature variations (i.e. large density variations) and gives reasonable results when confronted to the DNS/LES database with a priori tests. Moreover, although the current work only deals with one type of mixture (the species and the Prandtl number of the mixture are unchanged), the law-of-the-wall has been developed without any assumption concerning the species involved and the molecular Prandtl number. Thus this wall model should behave well with any other mixture.

\section{ACKNOWLEDGMENTS}

The authors are grateful to CNRS/DGA for funding this work and want to acknowledge the financial support and expertise of Snecma Propulsion Solide (SAFRAN group). We want to thank the CINES (Centre Informatique National pour l'Enseignement Supérieur) for the access to supercomputer facilities. J.M. Déoclézian is also gratefully acknowledged for fruitful discussions. 
[1] L. Prandtl. Über Flussigkeitsbewegung bei sehr kleiner Reibung. In Teubner, editor, Verhandlungen des dritten internationalen Mathematiker-Kongresses, pages 484-491, Leipzig, Germany, 1905.

[2] H. Blasius. Boundary layers in fluids with small friction. Z. Math. Phys., 56:1-37, 1908.

[3] T. von Kármán. Über laminare und turbulente Reibung. Z. Angew. Math. Mech., pages 233-252, 1921.

[4] T. von Kármán. Mechanische Ähnlichkeit und Turbulenz. Nach. Ges. Wiss. Göttingen, Math. Phys. Klasse, pages 58-68, 1930.

[5] F. H. Clauser. The turbulent boundary layer. Advances Appl. Mech., 4:1-51, 1956.

[6] S. V. Patankar and D. B. Spalding. Heat and mass transfer in boundary layers. MorganGrampian, London, 1967.

[7] C. A. Sleicher and M. W. Rouse. Convenient correlation for heat transfert to constant and variable property fluids in turbulent pipe flow. Int. J. Heat Mass Transfer, 18(5):677-683, May 1975.

[8] B. A. Kader. Temperature and concentration profiles in fully turbulent boundary layers. Int. J. Heat Mass Transfer, 24(9):1541-1544, 1981.

[9] H. Fernholz and P. Finley. A critical compilation of compressible turbulent boundary layer data. AGARD-AG, 223, 1977.

[10] P. Bradshaw. Compressible turbulent shear layers. Annu. Rev. Fluid Mech., 9:33-54, 1977.

[11] E. F. Spina, A. J. Smits, and S. K. Robinson. The physics of supersonic turbulent boundary layers. Annu. Rev. Fluid Mech., 26:287-319, 1994.

[12] G. N. Coleman, Kim J., and R. D. Moser. A numerical study of turbulent supersonic isothermal-wall channel flow. J. Fluid Mech., 305:159-183, 1995.

[13] P. G. Huang, G. N. Coleman, and P. Bradshaw. Compressible turbulent channel flows: DNS results and modelling. J. Fluid Mech., 305:185-218, 1995.

[14] P. Moin and K. Mahesh. Direct numerical simulation: a tool in turbulence research. Annu. Rev. Fluid Mech., 30:539-578, 1998.

[15] Y. Morinishi, S. Tamano, and K. Nakabayashi. Direct numerical simulation of compressible turbulent channel flow between adiabatic and isothermal walls. J. Fluid Mech., 502:273-308, 
2004 .

[16] P. Moin and J. Kim. Numerical investigation of turbulent channel flow. J. Fluid Mech., 118:341-377, 1982.

[17] S. Völker, R. D. Moser, and P. Venugopal. Optimal large eddy simulation of turbulent channel flow based on direct numerical simulation statistical data. Phys. Fluids, 14(10):3675-3691, October 2002.

[18] W. Wang and R. H. Pletcher. On the large eddy simulation of a turbulent channel flow with significant heat transfer. Phys. Fluids, 8(12):3354-3366, December 1996.

[19] L. D. Dailey, N. Meng, and R. H. Pletcher. Large eddy simulation of constant heat flux turbulent channel flow with property variations: quasi-developed model and mean flow results. J. Heat Trans., 125(1):27-38, February 2003.

[20] C. Brun, M. P. Boiarciuc, M. Haberkorn, and P. Comte. Larde eddy simulation of compressible channel flow. Theor. Comput. Fluid Dyn., 22(3-4):189-212, 2008.

[21] M. V. Morkovin. Effects of compressibility on turbulent flows. In A. Favre, editor, Méchanique de la Turbulence, pages 367-380. 1961.

[22] E. R. Van Driest. Turbulent boundary layer in compressible fluids. J. Aero. Sci, 18(3):145-160, 216, March 1951.

[23] E. R. Keener and E. J. Hopkins. Van Driest generalization applied to turbulent skin friction and velocity profiles measured on the wall of a Mach 7.4 wind tunnel. AIAA J., 11(12):17841785, December 1973.

[24] H. Fernholz and P. Finley. A critical commentary on mean flow data for two-dimensional compressible turbulent boundary layers. AGARD-AG, 253, 1980.

[25] P. G. Huang, P. Bradshaw, and T. J. Coakley. Skin friction and velocity profile family for compressible turbulent boundary layers. AIAA J., 31(9):1600-1604, September 1993.

[26] R. M. C. So, H. S. Zhang, T. B. Gatski, and C. G. Speziale. Logarithmic laws for compressible turbulent boundary layers. AIAA J., 32(11):2162-2168, November 1994.

[27] P. G. Huang and G. N. Coleman. Van driest transformation and compressible wall-bounded flows. AIAA J., 32(10):2110-2113, 1994.

[28] F. Nicoud. Numerical study of a channel flow with variable properties. In Annual Research Briefs, pages 289-310. Center for Turbulence Research, NASA Ames/Stanford Univ., 1998.

[29] M. P. Martín and G. V. Candler. DNS of a mach 4 boundary layer with chemical reactions. 
AIAA Paper 2000-0399, Jan. 2000.

[30] M. P. Martín and G. V. Candler. Temperature-fluctuation scaling in reacting boundary layers. In Annual Research Briefs, pages 151-162. Center for Turbulence Research, NASA Ames/Stanford Univ., 2001.

[31] J. Kim, P. Moin, and R. Moser. Turbulence statistics in fully developed channel flow at low Reynolds number. J. Fluid Mech., 177:133-166, 1987.

[32] R. K. Cheng and T. T. Ng. Some aspects of strongly heated turbulent boundary layer flow. Phys. Fluids, 25(8):1333-1341, August 1982.

[33] R.D. Moser, J Kim, and N.N. Mansour. Direct numerical simulation of turbulent channel flow up to $\mathrm{R}_{\tau}=590$. Phys. Fluids, 11(4):943-945, 1999.

[34] M. Teitel and R. A. Antonia. Heat transfer in fully developed turbulent channel flow: comparison between experiment and direct numerical simulations. Int. J. Heat Mass Transfer, 36(6):1701-1706, 1993.

[35] S. Hoyas and J. Jiménez. Scaling the velocity fluctuations in turbulent channel up to $\mathrm{R} e_{\tau}=$ 2003. Phys. Fluids, 18(011702):18-21, 2006.

[36] J. Jiménez and R. D. Moser. What are we learning from simulating wall turbulence? Phil. Trans. R. Soc. A, 365:715-732, 2007.

[37] B. Mohammadi and G. Puigt. Wall functions in computational fluid mechanics. Comput. Fluids, 35(10):1108-1115, 2006.

[38] R. L. Simpson. Characteristics of turbulent boundary layers at low Reynolds numbers with and without transpiration. J. Fluid Mech., 42(4):769-802, 1970.

[39] F. Nicoud and P. Bradshaw. A velocity transformation for heat and mass transfer. Phys. Fluids, 12(1):237-238, Jan. 2000.

[40] C. S. Subramanian and R. A. Antonia. Effect of Reynolds number on a slightly heated turbulent boundary layer. Int. J. Heat Mass Transfer, 24(11):1833-1846, 1981.

[41] Z. Han and R. D. Reitz. A temperature wall function formulation for variable-density turbulent flows with application to engine convective heat transfer modeling. Int. J. Heat Mass Transfer, 40(3):613-625, 1997.

[42] T. H. Shih, L. A. Povinelli, N. S. Liu, M. G. Potapczuk, and J. L. Lumley. A generalized wall function. NASA TM-1999-209398, July 1999.

[43] K. Suga, T. J. Craft, and H. Iacovides. An analytical wall-function for turbulent flows and 
heat transfer over rough walls. Int. J. Heat Fluid Flow, 27(5):852-866, 2006.

[44] P. G. Huang and P. Bradshaw. Law of the wall for turbulent flows in pressure gradients. AIAA J., 33(4):624-632, April 1995.

[45] M. Skote and D.S. Henningson. Analysis of the data base from a DNS of a separating turbulent boundary layer. In Annual Research Briefs, pages 225-237. Center for Turbulence Research, NASA Ames/Stanford Univ., 1999.

[46] M. Skote and D.S. Henningson. Direct numerical simulation of a separated turbulent boundary layer. J. Fluid Mech., 471:107-136, 2002.

[47] T. H. Shih, L. A. Povinelli, and N. S. Liu. Application of generalized wall function for complex turbulent flows. Journal of Turbulence, 4(15):1-16, April 2003.

[48] T.B. Nickels. Inner scaling for wall-bounded flows subject to large pressure gradients. J. Fluid Mech., 521:217-239, 2004.

[49] T. Houra and Y. Nagano. Effects of adverse pressure gradient on heat transfer mechanism in thermal boundary layer. Int. J. Heat Fluid Flow, 27:967-976, 2006.

[50] T. J. Craft, A. V. Gerasimov, H. Iacovides, and B. E. Launder. Progress in the generalization of wall-function treatments. Int. J. Heat Fluid Flow, 23(2):148-160, 2002.

[51] T. J. Craft, S. E. Gant, H. Iacovides, and B. E. Launder. A new wall function strategy for complex turbulent flows. Numer. Heat Transfer, 45(4):301-318, 2004.

[52] T. J. Craft, S. E. Gant, A. V. Gerasimov, H. Iacovides, and B. E. Launder. Development and application of wall-function treatments for turbulent forced and mixed convection flows. Fluid Dynamics Research, 38(2-3):127-144, 2006.

[53] J.M. Österlund, A. V. Johansson, H. M. Nagib, and M. H. Hites. A note on the overlap region in turbulent boundary layers. Phys. Fluids, 12(1):1-4, January 2000.

[54] M. H. Buschmann and M. Gad-el Hak. Debate concerning the mean-velocity profile of a turbulent boundary layer. AIAA J., 41(4):565-572, April 2003.

[55] M. H. Buschmann and M. Gad-el Hak. Structure of the canonical turbulent wall-bounded flow. AIAA J., 44(11):2500-2503, November 2006.

[56] P. A. Davidson, T. B. Nickels, and P. Å. Krogstad. The logarithmic structure function law in wall-layer turbulence. J. Fluid Mech., 550:51-60, 2006.

[57] M. H. Buschmann and M. Gad-el Hak. Generalized logarithmic law and its consequences. AIAA J., 41(1):40-48, January 2003. 
[58] S. V. Utyuzhnikov. Some new approaches to building and implementation of wall-functions for modeling of near-wall turbulent flows. Comput. Fluids, 34:771-784, 2005.

[59] K. K. Kuo. Principles of combustion. John Wiley \& Sons, Inc., Hoboken, New Jersey, 2nd edition, 2005.

[60] T. Poinsot and D. Veynante. Theoretical and Numerical Combustion. Edwards, 2nd edition, 2005.

[61] J. Amaya, O. Cabrit, D. Poitou, B. Cuenot, and M. El Hafi. Unsteady coupling of NavierStokes and radiative heat transfer solvers applied to an anisothermal multicomponent turbulent channel flow. In Proceedings of Eurotherm 83 - Computational Thermal Radiation in Participating Media III, Lisbon, Portugal, 15-17 April 2009.

[62] E. M. Sparrow, R. Eichhorn, and J. L. Gregg. Combined forced and free convection in a boundary layer flow. Phys. Fluids, 2(3):319-328, 1959.

[63] F. Nicoud, J. C. Traineau, and M. Prévost. Evolution of heat flux with mass flow rate in a subscale setup for the Ariane 5 solide rocket booster. In 2nd International Symposium on Engineering Turbulence Modelling and Measurements, Florence (Italy), May 31 - June 2, 1993.

[64] B. E. Poling, J. M. Prausnitz, and J. P. O'Connell. The properties of gases and liquids. McGraw-Hill, 5th edition, 2001.

[65] D. Y. Peng and D. B. Robinson. A new two-constant equation of state. Ind. Eng. Chem., Fundam., 15(1):59-64, 1976.

[66] J.O. Hirschfelder, F. Curtiss, and R.B. Bird. Molecular theory of gases and liquids. John Wiley \& Sons, 1964.

[67] V. Giovangigli. Multicomponent Flow Modeling. Birkhäuser, 1999.

[68] A. Ern and V. Giovangigli. Multicomponent transport algorithms. Lecture Notes in Physics, New Series Monographs, m 24. Springer-Verlag, Heidelberg, 1994.

[69] A. Ern and V. Giovangigli. Fast and accurate multicomponent transport property evaluation. J. Comput. Phys., 120(1):105-116, August 1995.

[70] J. Jiménez and P. Moin. The minimal flow unit in near-wall turbulence. J. Fluid Mech., 225:213-240, 1991.

[71] V. Moureau, G. Lartigue, Y. Sommerer, C. Angelberger, O. Colin, and T. Poinsot. Numerical methods for unsteady compressible multi-component reacting flows on fixed and moving grids. 
J. Comput. Phys., 202(2):710-736, 2005.

[72] P. Schmitt, T. Poinsot, B. Schuermans, and K. P. Geigle. Large-eddy simulation and experimental study of heat transfer, nitric oxide emissions and combustion instability in a swirled turbulent high-pressure burner. J. Fluid Mech., 570:17-46, 2007.

[73] S. Mendez and F. Nicoud. Large-eddy simulation of a bi-periodic turbulent flow with effusion. J. Fluid Mech., 598:27-65, 2008.

[74] O. Colin and M. Rudgyard. Development of high-order Taylor-Galerkin schemes for unsteady calculations. J. Comput. Phys., 162(2):338-371, 2000.

[75] F. Nicoud and F. Ducros. Subgrid-scale stress modelling based on the square of the velocity gradient tensor. Flow, Turb. and Combustion, 62:183-200, 1999.

[76] P. S. Andersen, W. M. Kays, and R. J. Moffat. Experimental results for the transpired turbulent boundary layer in an adverse pressure gradient. J. Fluid Mech., 69(2):353-375, 1975.

[77] W.M. Kays, M.E. Crawford, and B. Weigand. Convective Heat and Mass Transfer. McGrawHill, 2004.

[78] J. C. del Álamo and J. Jiménez. Spectra of the very large anisotropic scales in turbulent channels. Phys. Fluids, 15(6):L41-L44, 2003.

[79] J. C. del Álamo, J. Jiménez, P. Zandonade, and R. D. Moser. Scaling of the energy spectra of turbulent channels. J. Fluid Mech., 500:135-144, 2004.

[80] K.C. Kays. Turbulent Prandtl number. Where are we ? J. Heat Trans., 116:284-295, May 1994.

[81] B. E. Launder and D. B. Spalding. The numerical computation of turbulent flows. Comput. Methods Appl. Mech. Engng., 3(2):269-289, 1974. 